\title{
Overlapping Multi-Patch Structures in Isogeometric Analysis
}

\author{
S. Kargaran ${ }^{\mathrm{a}}$, B. Jüttler ${ }^{\mathrm{b}}$, S. K. Kleiss ${ }^{\mathrm{a}}$, A. Mantzaflaris ${ }^{\mathrm{a}, \mathrm{b}, \mathrm{c}}$, T. Takacs ${ }^{\mathrm{b}}$ \\ a Johann Radon Institute for Computational and Applied Mathematics (RICAM), Austrian Academy of Sciences, \\ Altenberger Straße 69, A-4040 Linz, Austria \\ ${ }^{b}$ Institute of Applied Geometry, Johannes Kepler University Linz, Altenberger Straße 69, A-4040 Linz, Austria \\ ${ }^{c}$ INRIA Sophia Antipolis, 2004 Route des Lucioles, 06902 Sophia Antipolis cedex, France
}

\begin{abstract}
In isogeometric analysis (IGA) the domain of interest is usually represented by B-spline or NURBS patches, as they are present in standard CAD models. Complex domains can often be represented as a union of simple overlapping subdomains, parameterized by (tensor-product) spline patches. Numerical simulation on such overlapping multi-patch domains is a serious challenge in IGA. To obtain non-overlapping subdomains one would usually reparameterize the domain or trim some of the patches. Alternatively, one may use methods that can handle overlapping subdomains.

In this paper, we propose a non-iterative, robust and efficient method defined directly on overlapping multi-patch domains. Consequently, the problem is divided into several sub-problems, which are coupled in an appropriate way. The resulting system can be solved directly in a single step. We compare the proposed method with iterative Schwarz domain decomposition approaches and observe that our method reduces the computational cost significantly, especially when handling subdomains with small overlaps.

Summing up, our method significantly simplifies the domain parameterization problem, since we can represent any domain of interest as a union of overlapping patches without the need to introduce trimming curves/surfaces. The performance of the proposed method is demonstrated by several numerical experiments for the Poisson problem and linear elasticity in two and three dimensions.
\end{abstract}

Keywords: isogeometric analysis, coupling, multi-patch, overlaps, Schwarz method

\section{Introduction}

Isogeometric analysis (IGA) is a computational approach, introduced by Hughes et al. [1]. IGA connects the technology of computer aided design (CAD) with numerical simulation via finite element analysis, e.g., [2, 3, 4]. In IGA, the same basis functions (typically tensor-product B-splines) are used for describing the geometry as well as for the numerical analysis. Hence, simulations can be performed directly on the geometry representation of CAD models. 
In CAD a domain of interest is usually represented by a collection of curves in $2 \mathrm{D}$ or surfaces in 3D describing its boundary. The design of complex shapes in CAD systems is often based on Boolean operations applied to simple objects, such as tensor-product NURBS patches. Hence, the resulting boundary curves or surfaces are trimmed NURBS patches, see e.g. [5]. These patches use only certain parts of the full tensor product domains, due to additional geometric boundaries specified by trimming curves or surfaces.

If the domain of interest is constructed only by Boolean unions, as in Figure 1, then the domain can be parameterized by trimmed (center) or overlapping (right) tensor-product patches.
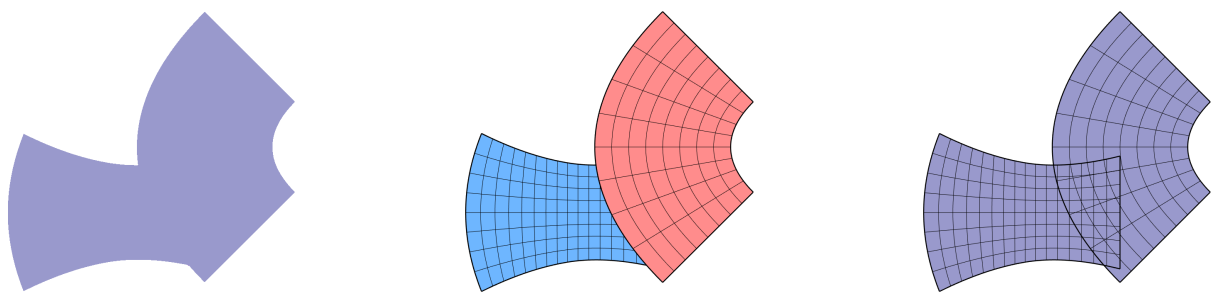

Figure 1: A non-four sided domain (left) can be represented either by non-overlapping, but trimmed, patches (center), or by untrimmed, but overlapping, patches (right).

Having given only a boundary representation one can obtain a collection of tensor-product patches covering the whole domain. It is relatively easy to construct such a covering if all boundary patches are untrimmed and overlaps are allowed.

However, in 3D CAD, the boundary representation often consists of a collection of trimmed NURBS patches, which cannot be represented as an overlapping multi-patch domain directly, e.g., if the domain is given as a Boolean intersection of two patches.

Alternatively, one may be provided with or construct a parameterization of the domain with non-overlapping patches. In that case patches may share interfaces. Performing numerical simulations on such multi-patch domains is not straightforward since one needs to couple the patch-wise discretizations in an appropriate way across the interfaces or on the overlap regions.

There exist several approaches for solving a PDE on multi-patch domains that consist of mutually disjoint patches. For matching or nested interfaces, the smoothness of the solution across patch boundaries can be enforced strongly, simply by identifying the degrees of freedom on the interfaces between the subdomains $[6,7,8]$. Depending on the order of the considered PDE, a higher order of continuity may be needed $[9,10,11]$.

The situation becomes more complicated if non-matching interfaces are present. In this case, the coupling constraints need to be enforced weakly. The mortar method [12] can be employed to solve a PDE on the non-overlapping subdomains. Here, the continuity conditions are imposed using Lagrange multipliers. Similarly, in $[13,14]$, the authors propose the INTERNODES method to couple Galerkin discretizations for non-overlapping subdomains. Instead of Lagrange multipliers, an 
interpolation scheme is used to enforce the continuity constraints on the non-matching interfaces. Alternatively, the Nitsche method can be used to impose weak coupling conditions on the nonmatching interfaces [15, 16, 17, 18, 19], similar to discontinuous Galerkin methods [20, 21, 22].

Several techniques have been developed that deal directly with trimmed domain parameterizations in IGA, see e.g. [23, 24, 25] as well as the survey paper [5] and the references cited therein. Applying IGA to trimmed domains creates three major challenges.

The first one is related to the stability of the basis functions near the trimmed boundary. Some of these basis functions, possessing supports that are cut by the geometric boundary, are not stable since their remaining support is very small. One way to resolve this issue is to add these functions to nearby stable basis functions. This concept of extended B-splines was introduced in [26]. The resulting modification of unstable basis functions is considered in [27, 28] to introduce the immersed and extended B-spline basis functions respectively.

The second challenge is to devise efficient methods for numerical quadrature that are able to deal with functions defined on trimmed domains. Since numerical quadrature rules on boxes are easy to generate, it has been proposed in e.g. [29, 18, 30] to employ finer cells near the trimming boundary. Alternatively, mesh deformation techniques can be applied, as in [31], in order to extend the quadrature rules from simple boxes to more general, deformed elements. The accuracy of adaptive quadrature rule is improved in [32], where the authors define accurate and efficient quadrature rules on general trimmed elements, that are local and unique to the element. The recent preprint [33] establishes special quadrature rules for trimmed geometries based on expansions of the integrands that are involved.

Finally, the coupling of adjacent trimmed domains needs to be dealt with. Most standard methods for coupling patch-wise discretizations are not applicable if trimmed patches are present. Notable exceptions are [34], based on isogeometric B-rep elements, Nitsche-based methods, such as [18], or discontinuous Galerkin-like approaches. The latter ones can be adapted to deal with overlapping subdomains directly as in [35].

Alternatively, one can convert the trimmed patches into untrimmed patches by suitably splitting them and generating new parameterizations as presented in [36]. For instance, triangular Bézier or Coons patches are used to convert a trimmed model to a watertight representation, see [37, 38]. However, the reparameterization is costly and may increase the number of patches substantially. Also, it is not guaranteed to preserve the exactness of the geometry used for the simulation, which is considered to be one of the main assets of the isogeometric framework.

Summing up, the presence of trimmed elements is a source of considerable difficulties. Several techniques have been developed to deal with them, which are based on different approaches, such as geometry preprocessing or Nitsche-based methods. In particular, we note that the proposed geometry processing (splitting the domain into single patches, followed by a reparameterization) is not in line with the isogeometric paradigm of exact geometry representation. It may also lead to a large number of small subdomains. 
In many cases, the use of trimming can be avoided by considering overlapping domains, see Fig. 1. Numerical simulation on domains of this type can be performed with the help of overlapping domain decomposition methods, see [39] and the references therein. Using the framework of isogeometric analysis, both additive Schwarz (ADS) and multiplicative Schwarz (MPS) domain decomposition methods, which are iterative approaches, have been explored in [40]. In each iteration step, the PDE is solved separately on each subdomain. The sub-problems are coupled by imposing boundary conditions on the boundary of the overlap regions. Schwarz-type methods become costly for a large number of overlapping subdomains and require a high number of iterations if small overlaps are present.

In order to perform numerical simulations on multi-patch domains with overlaps, we propose in this paper the overlapping multi-patch (OMP) method, which is a non-iterative reformulation of the Schwarz method. More precisely, we show how to perform isogeometric simulations on overlapping multi-patch structures by numerically solving a single system. This system is derived by introducing suitable extension operators, which are used to couple the solutions on the individual subdomains.

For the Poisson equation, which serves as a model problem, we derive an equivalent reformulation in the continuous case, and we present numerical results which indicate that the isogeometric discretization preserves the coercivity for a particular choice of the extension operator.

We also use numerical experiments to compare the computational costs of the OMP method with the ADS and MPS methods. According to these results, the non-iterative reformulation provides a significant reduction of the overall costs and is far more robust with respect to the size of the overlaps.

The remainder of the paper is organized as follows. Section 2 introduces the overlapping multipatch formulation, and shows that it is equivalent to the original one in the continuous case. The next section describes the isogeometric discretization via B-splines. We also set up the final system according to different extension operators. The existence and uniqueness of the discretized problem is studied via numerical experiments in Section 4, and Section 5 investigates the performance of the OMP method on different overlapping multi-patch domains. Finally we conclude the paper in Section 6.

\section{The overlapping multi-patch formulation}

In this section we introduce the geometry representation that we consider and derive the overlapping multi-patch formulation on a simplified model problem.

As a model problem we consider in Section 2.1 the Poisson problem on an open and bounded domain $\Omega \subset \mathbb{R}^{d}$ with a given right-hand side $f$, and zero Dirichlet boundary conditions. The approach we present in the following is not restricted to this specific problem. In Section 2.3 we formulate all definitions and results with respect to the Poisson problem. However, the method can be applied to other second order elliptic problems or other boundary conditions as well. 
The physical domain $\Omega$ is formed by overlapping subdomains. We assume that the subdomains are open and that they only have pairwise overlaps, the intersection of three or more subdomains is always empty. Also, we do not allow interfaces between subdomains. It means

$$
\Omega^{i} \cap \Omega^{j} \cap \Omega^{k}=\emptyset \text { and } \overline{\Omega^{i} \cap \Omega^{j}}=\overline{\Omega^{i}} \cap \overline{\Omega^{j}}, \quad \forall i \neq j \neq k \neq i .
$$

In order to keep the presentation simple, we introduce the formulation for two patches $\Omega^{1}$ and $\Omega^{2}$ only. However, the approach works also for multiple patches, and in Section 5 we present some examples of domains having more than two patches with pairwise overlap.

\subsection{Model problem}

Assume that a physical domain $\Omega \subset \mathbb{R}^{d}$ is given. We consider the two bounded forms:

$$
a(u, v)=\int_{\Omega} \nabla u \nabla v \mathrm{~d} \boldsymbol{\xi} \text { and } \quad \ell(v)=\int_{\Omega} f v \mathrm{~d} \boldsymbol{\xi}
$$

then the weak form of the Poisson problem can be formulated as follows

Problem 1. Find $u \in H_{0}^{1}(\Omega)$, such that

$$
a(u, v)=\ell(v) \quad \text { for all } v \in H_{0}^{1}(\Omega) .
$$

In the following we define a formulation on overlapping multi-patch structures. This formulation will turn out to be equivalent to the initial Problem 1.

\subsection{The overlapping multi-patch structure}

We are interested in domains

$$
\Omega=\Omega^{1} \cup \Omega^{2},
$$

created by forming the union of two open subdomains with non-empty overlap

$$
\Phi^{12}=\Omega^{1} \cap \Omega^{2} \neq \emptyset,
$$

see Fig. 2. The boundary of the two subdomains is subdivided into the Dirichlet and coupling boundary

$$
\Gamma_{D}^{k}=\partial \Omega^{k} \cap \partial \Omega \quad \text { and } \quad \Gamma_{C}^{k}=\partial \Omega^{k} \cap \Omega^{\circ} \quad(\text { for } k \in\{1,2\}),
$$

respectively. Here $\Omega^{\circ}$ denotes the interior of the domain $\Omega$.

In order to exploit the special structure of the domain, we introduce the extension operators

$$
M^{k}: H^{1}\left(\Omega^{k^{\prime}}\right) \rightarrow H^{1}\left(\Omega^{k}\right),
$$




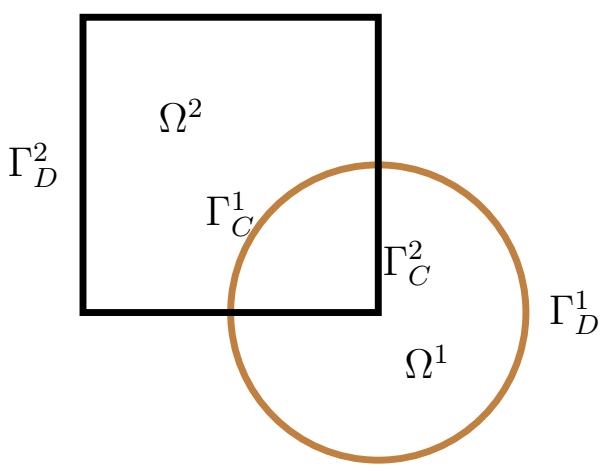

Figure 2: Two overlapping patches.

which take a function from one patch and map it to the other. Here, for $k \in\{1,2\}, k^{\prime}$ is defined as the other index, i.e., $k^{\prime}=3-k$. We will use this notation throughout. Note that the image of the projector has non-vanishing trace at the coupling boundary. The projector $M^{k}$ is bounded and defined to take the value of a function on $\Omega^{k^{\prime}}$ at the coupling boundary $\Gamma_{C}^{k}$ and extend it into the patch $\Omega^{k}$. More precisely, these operators are assumed to satisfy the conditions

$$
M^{k} v= \begin{cases}v & \text { on } \Gamma_{C}^{k} \\ 0 & \text { on } \Gamma_{D}^{k}\end{cases}
$$

Clearly, there are several possibilities to choose these operators, which will be discussed in more detail later.

\subsection{The overlapping multi-patch problem}

To define the local problems, we consider the restrictions of the two forms $a(u, v)$ and $\ell(v)$ in (1) to the two subdomains,

$$
a^{k}\left(u^{k}, v^{k}\right)=\int_{\Omega^{k}} \nabla u^{k} \nabla v^{k} \mathrm{~d} \xi \quad \text { and } \quad \ell^{k}\left(v^{k}\right)=\int_{\Omega^{k}} f v^{k} \mathrm{~d} \xi,
$$

which are defined for $u^{k}, v^{k} \in H^{1}\left(\Omega^{k}\right)$. For $\left(u^{1}, u^{2}\right),\left(v^{1}, v^{2}\right) \in H^{1}\left(\Omega^{1}\right) \times H^{1}\left(\Omega^{2}\right)$, we define

$$
\mathcal{A}\left(\left(u^{1}, u^{2}\right),\left(v^{1}, v^{2}\right)\right)=a^{1}\left(u^{1}, v^{1}\right)+a^{2}\left(u^{2}, v^{2}\right)
$$

and

$$
\mathcal{L}\left(v^{1}, v^{2}\right)=\ell^{1}\left(v^{1}\right)+\ell^{2}\left(v^{2}\right) .
$$

In the following we will introduce functions $u_{0}^{k} \in H_{0}^{1}\left(\Omega^{k}\right)$, satisfying

$$
u^{k}=u_{0}^{k}+M^{k} u^{k^{\prime}} .
$$


In order for this equation to be solvable, i.e.,

$$
\left(\begin{array}{l}
u^{1} \\
u^{2}
\end{array}\right)=\left(\begin{array}{cc}
I^{1} & -M^{1} \\
-M^{2} & I^{2}
\end{array}\right)^{-1}\left(\begin{array}{l}
u_{0}^{1} \\
u_{0}^{2}
\end{array}\right),
$$

we need the following assumption.

Assumption 1. The operators $M^{1} M^{2}: H^{1}\left(\Omega^{1}\right) \rightarrow H^{1}\left(\Omega^{1}\right)$ and $M^{2} M^{1}: H^{1}\left(\Omega^{2}\right) \rightarrow H^{1}\left(\Omega^{2}\right)$ are contractions.

Since $M^{k} M^{k^{\prime}}$ is a contraction, we can define $M^{k k^{\prime}}: H^{1}\left(\Omega^{k}\right) \rightarrow H^{1}\left(\Omega^{k}\right)$ as

$$
M^{k k^{\prime}}=\sum_{\ell=0}^{\infty}\left(M^{k} M^{k^{\prime}}\right)^{\ell}=\left(I^{k}-M^{k} M^{k^{\prime}}\right)^{-1},
$$

where $I^{k}$ is the identity operator mapping $H^{1}\left(\Omega^{k}\right)$ onto itself. A sufficient condition for Assumption 1 is

$$
M^{k} M^{k^{\prime}} u^{k} \equiv 0 \quad \text { for all } u^{k} \in H^{1}\left(\Omega^{k}\right) \text { and } k \in\{1,2\} .
$$

Moreover, we assume that the operator $M^{k}$ depends only on the value of the function in the overlap region.

Assumption 2. The operator $M^{k}$ satisfies that for all $u^{k^{\prime}} \in H^{1}\left(\Omega^{k^{\prime}}\right), M^{k} u^{k^{\prime}} \equiv 0$ if $u^{k^{\prime}}=0$ on $\Phi^{12}$.

Remark 1. In the discrete setting we define operators that satisfy a stronger version of Assumption 2. More precisely, we have $M^{k} u^{k^{\prime}} \equiv 0$, if $u^{k^{\prime}}=0$ on $\Gamma_{C}^{k}$.

Now we can formulate the localized weak form of the model problem:

Problem 2. Find $\left(u_{0}^{1}, u_{0}^{2}\right) \in H_{0}^{1}\left(\Omega^{1}\right) \times H_{0}^{1}\left(\Omega^{2}\right)$, such that

$$
\mathcal{A}\left(\left(u^{1}, u^{2}\right),\left(v_{0}^{1}, v_{0}^{2}\right)\right)=\mathcal{L}\left(v_{0}^{1}, v_{0}^{2}\right) \quad \text { for all }\left(v_{0}^{1}, v_{0}^{2}\right) \in H_{0}^{1}\left(\Omega^{1}\right) \times H_{0}^{1}\left(\Omega^{2}\right),
$$

where

$$
u^{k}=M^{k k^{\prime}}\left(u_{0}^{k}+M^{k} u_{0}^{k^{\prime}}\right) \quad \text { for } k \in\{1,2\} .
$$

We can show that this form of the problem is equivalent to the original one:

Theorem 1. Consider functions $u \in H_{0}^{1}(\Omega)$ and $\left(u_{0}^{1}, u_{0}^{2}\right) \in H_{0}^{1}\left(\Omega^{1}\right) \times H_{0}^{1}\left(\Omega^{2}\right)$.

(i) If $u$ is a solution of Problem 1, then $\left(\left.u\right|_{\Omega^{1}}-M^{1}\left(\left.u\right|_{\Omega^{2}}\right),\left.u\right|_{\Omega^{2}}-M^{2}\left(\left.u\right|_{\Omega^{1}}\right)\right)$ is a solution of Problem 2. 
(ii) If $\left(u_{0}^{1}, u_{0}^{2}\right)$ is a solution of Problem 2, then

$$
u= \begin{cases}M^{12}\left(u_{0}^{1}+M^{1} u_{0}^{2}\right) & \text { on } \Omega^{1} \\ M^{21}\left(u_{0}^{2}+M^{2} u_{0}^{1}\right) & \text { on } \Omega^{2} \backslash \Phi^{12}\end{cases}
$$

is a solution of Problem 1. The solution of Problem 2 is unique.

Proof. See Appendix A.

For 1D domains and a suitable choice of extension operators, we can moreover prove that Problem 2 satisfies the assumptions of the Lax-Milgram lemma, i.e., the bilinear and linear forms are bounded and the bilinear form is coercive.

Lemma 1. Assume that $\Omega=\Omega^{1} \cup \Omega^{2} \subset \mathbb{R}$, with $\left.\Omega^{1}=\right] a_{1}, b_{1}\left[\right.$ and $\left.\Omega^{2}=\right] a_{2}, b_{2}[$. There exist constants $\bar{\alpha}, \underline{\alpha}$ and $\Lambda$, depending on the relative size of the overlap, such that

- $\left|\mathcal{A}\left(\left(u^{1}, u^{2}\right),\left(v_{0}^{1}, v_{0}^{2}\right)\right)\right| \leq \bar{\alpha}\left\|\left(u_{0}^{1}, u_{0}^{2}\right)\right\|_{H^{1}\left(\Omega^{1}\right) \times H^{1}\left(\Omega^{2}\right)}\left\|\left(v_{0}^{1}, v_{0}^{2}\right)\right\|_{H^{1}\left(\Omega^{1}\right) \times H^{1}\left(\Omega^{2}\right)}$,

- $\mathcal{A}\left(\left(u^{1}, u^{2}\right),\left(u_{0}^{1}, u_{0}^{2}\right)\right) \geq \underline{\alpha}\left\|\left(u_{0}^{1}, u_{0}^{2}\right)\right\|_{H^{1}\left(\Omega^{1}\right) \times H^{1}\left(\Omega^{2}\right)}^{2}$,

- $\left|\mathcal{L}\left(\left(v_{0}^{1}, v_{0}^{2}\right)\right)\right| \leq \Lambda\left\|\left(v_{0}^{1}, v_{0}^{2}\right)\right\|_{H^{1}\left(\Omega^{1}\right) \times H^{1}\left(\Omega^{2}\right)}$,

for the extension operators specified in (B.1). Here, all functions $u^{k}, u_{0}^{k}$ and $v_{0}^{k}(k \in\{1,2\})$ are given as in Problem 2. The norm is given as $\left\|\left(v^{1}, v^{2}\right)\right\|_{H^{1}\left(\Omega^{1}\right) \times H^{1}\left(\Omega^{2}\right)}=\left(\left\|v^{1}\right\|_{H^{1}\left(\Omega^{1}\right)}^{2}+\left\|v^{2}\right\|_{H^{1}\left(\Omega^{2}\right)}^{2}\right)^{\frac{1}{2}}$.

Proof. See Appendix B.

Remark 2. Note that the proof is based on harmonic extension operators, i.e., operators extending the function values on the coupling boundary into the overlap $\Omega^{1} \cap \Omega^{2}$ such that the resulting function is harmonic. A generalization of the approach to higher dimensions, using harmonic extension operators, may be possible. Moreover, the proof of Lemma 1 motivates the use of a quasi-harmonic extension operator that we will define in Section 3.4 in the discrete setting.

Problem 2 can be rewritten as follows.

Problem 3. Find $\left(u_{0}^{1}, u_{0}^{2}\right) \in H_{0}^{1}\left(\Omega^{1}\right) \times H_{0}^{1}\left(\Omega^{2}\right)$ and $\left(u_{M}^{1}, u_{M}^{2}\right) \in H^{1}\left(\Omega^{1}\right) \times H^{1}\left(\Omega^{2}\right)$ such that

$$
\begin{aligned}
\mathcal{A}\left(\left(u_{0}^{1}+u_{M}^{1}, u_{0}^{2}+u_{M}^{2}\right),\left(v_{0}^{1}, v_{0}^{2}\right)\right) & =\mathcal{L}\left(v_{0}^{1}, v_{0}^{2}\right), \quad \forall\left(v_{0}^{1}, v_{0}^{2}\right) \in H_{0}^{1}\left(\Omega^{1}\right) \times H_{0}^{1}\left(\Omega^{2}\right) \\
u_{M}^{1}-M^{1}\left(u_{0}^{2}+u_{M}^{2}\right) & \equiv 0 \\
u_{M}^{2}-M^{2}\left(u_{0}^{1}+u_{M}^{1}\right) & \equiv 0 .
\end{aligned}
$$


In the formulation of Problem 3, we replace $M^{k} u^{k^{\prime}}$ with $u_{M}^{k}$ and obtain the additional constraints from $u^{k}=u_{0}^{k}+M^{k} u^{k^{\prime}}$. This is the problem that we want to study and discretize in the following. For the variational equation (11) we will use a standard Galerkin discretization. The two equations (12) and (13), which implicitly define the extension operators, are discretized using finite differences as well as a collocation scheme at the coupling boundary.

\section{Isogeometric discretization}

In this section we briefly introduce B-spline basis functions and the local geometry mappings (see also [2]). To represent the discrete solutions we define the spaces on each subdomain by selecting the corresponding basis functions.

\subsection{Geometry mapping}

Let $\mathcal{S}_{\Xi}^{p}:(0,1) \rightarrow \mathbb{R}$ be a univariate B-spline space of degree $p$ for a suitable open knot vector $\Xi$. Let $\hat{\beta}_{i, p}$, for $i=1, \ldots, n$, be the standard basis of the B-spline space $\mathcal{S}_{\Xi}^{p}$ (see, [41]).

For each patch $\Omega^{k}$ and each direction $\xi_{\nu}$ we have given a separate B-spline space $\mathcal{S}_{\Xi_{\nu}^{k}}^{p}$ and corresponding B-spline basis functions $\hat{\beta}_{i_{\nu}}^{k, \nu}$, with $1 \leq i_{\nu} \leq n_{\nu}^{k}$ for $k \in\{1,2\}$. We then define the tensor-product B-spline space $\mathcal{S}^{k}=\mathcal{S}_{\Xi_{1}^{k}}^{p} \otimes \ldots \otimes \mathcal{S}_{\Xi_{d}^{k}}^{p}$ with the basis functions

$$
\hat{\beta}_{i}^{k}\left(\xi_{1}, \ldots, \xi_{d}\right)=\hat{\beta}_{i_{1}}^{k, 1}\left(\xi_{1}\right) \cdot \ldots \cdot \hat{\beta}_{i_{d}}^{k, d}\left(\xi_{d}\right),
$$

where the index $i \in \mathcal{I}^{k}$ corresponds to the tuple $\left(i_{1}, \ldots, i_{d}\right)$. So $\mathcal{I}^{k}$ is the index set

$$
\left\{\left(i_{1}, \ldots, i_{d}\right) \in \mathbb{Z}^{d}: 1 \leq i_{\nu} \leq n_{\nu}^{k} \text { for all } \nu=1, \ldots, d\right\} .
$$

We assume that the physical domains $\Omega^{k}$ are images of spline mappings $\mathbf{G}^{k} \in\left(\mathcal{S}^{k}\right)^{d}$, i.e., $\Omega^{k}=$ $\mathbf{G}^{k}\left(\hat{\Omega}^{k}\right)$ for $k=1,2$, and $\hat{\Omega}^{k}=(0,1)^{d}$ are the corresponding parametric domains.

\subsection{Isogeometric spaces}

We can now define the isogeometric space on every patch as

$$
V_{h}^{k}=\operatorname{span}\left\{\beta_{i}^{k}=\hat{\beta}_{i}^{k} \circ\left(\mathbf{G}^{k}\right)^{-1} \mid i \in \mathcal{I}^{k}\right\}
$$

for $k \in\{1,2\}$.

For every patch $\Omega^{k}$ we now define three subspaces of the full space $V_{h}^{k}$ and corresponding index sets as follows:

- Interior functions:

$$
V_{0 h}^{k}=\operatorname{span}\left\{\beta_{i}^{k} \mid i \in \mathcal{I}_{0}^{k}\right\}=V_{h}^{k} \cap H_{0}^{1}\left(\Omega^{k}\right), \text { with } \mathcal{I}_{0}^{k}=\left\{i \in \mathcal{I}^{k} \mid \beta_{i}^{k}=0 \text { on } \partial \Omega^{k}\right\},
$$


- Coupling overlap functions:

$$
V_{c h}^{k}=\operatorname{span}\left\{\beta_{i}^{k} \mid i \in \mathcal{I}_{c}^{k}\right\} \subset H^{1}\left(\Omega^{k}\right) \text {, with } \mathcal{I}_{c}^{k}=\left\{i \in \mathcal{I}^{k} \mid \operatorname{supp} \beta_{i}^{k} \cap \Gamma_{C}^{k} \neq \emptyset\right\},
$$

- Non-coupling overlap functions:

$$
V_{\Phi h}^{k}=\operatorname{span}\left\{\beta_{i}^{k} \mid i \in \mathcal{I}_{\Phi}^{k}\right\} \subset V_{0 h}^{k} \subset H_{0}^{1}\left(\Omega^{k}\right), \text { with } \mathcal{I}_{\Phi}^{k}=\left\{i \in \mathcal{I}^{k} \mid \operatorname{supp} \beta_{i}^{k} \subset \Phi^{12}\right\} .
$$

Let moreover $V_{\Phi c h}^{k}=V_{c h}^{k} \oplus V_{\Phi h}^{k}$ and $\mathcal{I}_{\Phi c}^{k}=\mathcal{I}_{c}^{k} \cup \mathcal{I}_{\Phi}^{k}$. We define the descretized space

$$
V_{0 h}=V_{0 h}^{1} \times V_{0 h}^{2} \subset H_{0}^{1}\left(\Omega^{1}\right) \times H_{0}^{1}\left(\Omega^{2}\right),
$$

which is used both as the trial function space for $\left(u_{0 h}^{1}, u_{0 h}^{2}\right)$ and as the test function space.

\subsection{The discretized problem}

Using the spaces defined in the previous section, Problem 3 can be discretized as follows.

Problem 4. Find $\left(u_{0 h}^{1}, u_{0 h}^{2}\right) \in V_{0 h}$ and $\left(u_{M h}^{1}, u_{M h}^{2}\right) \in V_{\Phi c h}^{1} \times V_{\Phi c h}^{2}$ such that

$$
\begin{array}{rlrl}
\left.\mathcal{A}\left(u_{0 h}^{1}+u_{M h}^{1}, u_{0 h}^{2}+u_{M h}^{2}\right),\left(v_{0 h}^{1}, v_{0 h}^{2}\right)\right) & =\mathcal{L}\left(v_{0 h}^{1}, v_{0 h}^{2}\right) & \forall\left(v_{0 h}^{1}, v_{0 h}^{2}\right) \in V_{0 h} \\
u_{M h}^{1}-M_{h}^{1}\left(u_{0 h}^{2}+u_{M h}^{2}\right) & =0 & \text { on } \Omega^{1} & \\
u_{M h}^{2}-M_{h}^{2}\left(u_{0 h}^{1}+u_{M h}^{1}\right) & =0 & \text { on } \Omega^{2}, &
\end{array}
$$

where $M_{h}^{1}$ and $M_{h}^{2}$ are suitable discretizations of the operators $M^{1}$ and $M^{2}$, respectively, which will be defined in Subsection 3.4.

Remark 3. Note that Problem 4 can be solved with non-zero Dirichlet boundary conditions as well.

Remark 4. For 1D domains, having a bounded and coercive bilinear form due to Lemma 1, we can apply Céa's lemma and obtain an estimate of the form

$$
\left\|\left(u_{0}^{1}-u_{0 h}^{1}, u_{0}^{2}-u_{0 h}^{2}\right)\right\|_{H^{1}\left(\Omega^{1}\right) \times H^{1}\left(\Omega^{2}\right)} \leq \frac{\bar{\alpha}}{\underline{\alpha}} \inf _{\left(v_{0 h}^{1}, v_{0 h}^{2}\right) \in V_{0 h}}\left\|\left(u_{0}^{1}-v_{0 h}^{1}, u_{0}^{2}-v_{0 h}^{2}\right)\right\|_{H^{1}\left(\Omega^{1}\right) \times H^{1}\left(\Omega^{2}\right)},
$$

where the right hand side can be bounded using standard spline approximation estimates. Hence, in $1 \mathrm{D}$, optimal convergence in the energy norm follows directly. Note that, by definition, the error contribution is measured twice in the overlap region. A more rigorous analysis remains future work. 
The functions in Problem 4 can be expressed as

$$
u_{0 h}^{k}(\mathbf{x})=\sum_{i \in \mathcal{I}_{0}^{k}} c_{i}^{k} \beta_{i}^{k}(\mathbf{x}), \quad u_{M h}^{k}(\mathbf{x})=\sum_{i \in \mathcal{I}_{\Phi c}^{k}} d_{i}^{k} \beta_{i}^{k}(\mathbf{x})
$$

and

$$
u_{h}^{k}(\mathbf{x})=u_{0 h}^{k}(\mathbf{x})+u_{M h}^{k}(\mathbf{x})
$$

for $\mathbf{x}=\left(x_{1}, \ldots, x_{d}\right) \in \Omega^{k}$. The variational equation (16) in Problem 4 is equivalent to

$$
\begin{aligned}
& \sum_{i \in \mathcal{I}_{0}^{1}} c_{i}^{1} \underbrace{\int_{\Omega^{1}} \nabla \beta_{i}^{1} \nabla \beta_{j}^{1} \mathrm{~d} \mathbf{x}}_{=: K_{j i}^{1}}+\sum_{i \in \mathcal{I}_{\Phi c}^{1}} d_{i}^{1} \underbrace{\int_{\Omega^{1}} \nabla \beta_{i}^{1} \nabla \beta_{j}^{1} \mathrm{~d} \mathbf{x}}_{=K_{j i}^{1}}=\underbrace{\int_{\Omega^{1}} f \beta_{j}^{1} \mathrm{~d} \mathbf{x}}_{=: f_{j}^{1}} \quad \forall j \in \mathcal{I}_{0}^{1} \\
& \sum_{i \in \mathcal{I}_{0}^{2}} c_{i}^{2} \underbrace{\int_{\Omega^{2}} \nabla \beta_{i}^{2} \nabla \beta_{j}^{2} \mathrm{~d} \mathbf{x}}_{=: K_{j i}^{2}}+\sum_{i \in \mathcal{I}_{\Phi c}^{2}} d_{i}^{2} \underbrace{\int_{\Omega^{2}} \nabla \beta_{i}^{2} \nabla \beta_{j}^{2} \mathrm{~d}}_{=K_{j i}^{2}} \mathbf{x}=\underbrace{\int_{\Omega^{2}} f \beta_{j}^{2} \mathrm{~d} \mathbf{x}}_{=: f_{j}^{2}}, \quad \forall j \in \mathcal{I}_{0}^{2} .
\end{aligned}
$$

Since $\mathcal{I}_{\Phi c}^{k}=\mathcal{I}_{c}^{k} \cup \mathcal{I}_{\Phi}^{k}$, we can write

$$
\begin{array}{ll}
\sum_{i \in \mathcal{I}_{0}^{1}} K_{j i}^{1} c_{i}^{1}+\sum_{i \in \mathcal{I}_{c}^{1}} K_{j i}^{1} d_{i}^{1}+\sum_{i \in \mathcal{I}_{\Phi}^{1}} K_{j i}^{1} d_{i}^{1}=f_{j}^{1} & \forall j \in \mathcal{I}_{0}^{1} \\
\sum_{i \in \mathcal{I}_{0}^{2}} K_{j i}^{2} c_{i}^{2}+\sum_{i \in \mathcal{I}_{c}^{2}} K_{j i}^{2} d_{i}^{2}+\sum_{i \in \mathcal{I}_{\Phi}^{2}} K_{j i}^{2} d_{i}^{2}=f_{j}^{2}, & \forall j \in \mathcal{I}_{0}^{2} .
\end{array}
$$

We can write the matrices and vectors in block form as

$$
\left(\begin{array}{cccccc}
K_{0}^{1} & 0 & K_{c}^{1} & 0 & K_{\Phi}^{1} & 0 \\
0 & K_{0}^{2} & 0 & K_{c}^{2} & 0 & K_{\Phi}^{2}
\end{array}\right)\left(\begin{array}{c}
\mathbf{c}_{0}^{1} \\
\mathbf{c}_{0}^{2} \\
\mathbf{d}_{c}^{1} \\
\mathbf{d}_{c}^{2} \\
\mathbf{d}_{\Phi}^{1} \\
\mathbf{d}_{\Phi}^{2}
\end{array}\right)=\left(\begin{array}{c}
\mathbf{f}^{1} \\
\mathbf{f}^{2}
\end{array}\right)
$$

where the block matrix is composed of sub-matrices $K_{*}^{k}=\left(K_{j i}^{k}\right)_{i \in \mathcal{I}_{*}^{k}, j \in \mathcal{I}_{0}^{k}}$, with $* \in\{0, c, \Phi\}$, and the vectors of coefficients are given as $\mathbf{c}_{0}^{k}=\left(c_{i}^{k}\right)_{i \in \mathcal{I}_{0}^{k}}$ and $\mathbf{d}_{*}^{k}=\left(d_{i}^{k}\right)_{i \in \mathcal{I}_{*}^{k}}$, with $* \in\{c, \Phi\}$.

\subsection{Discrete extension operators}

In the following we consider the discretization of equations (17)-(18). 
The continuous extension operators have only to satisfy the condition (4). For the discrete operators we assume $\operatorname{Im}\left(M_{h}^{k}\right) \subseteq V_{\Phi c h}^{k}$. We call $M_{h}^{k}$ a minimal extension operator, if $M_{h}^{k}: V_{0 h}^{k^{\prime}} \rightarrow$ $V_{c h}^{k}$. There are different possible choices of minimal extension operators, which depend on the computation of the degrees of freedom related to the boundary of the overlap region.

As in the continuous case, we want the discrete extension operators to satisfy the following equation defined on the boundary of the overlap $\Phi^{12}$

$$
\left(M_{h}^{k} u_{h}^{k^{\prime}}\right)(\mathbf{x})=u_{M h}^{k}(\mathbf{x}) \approx u_{h}^{k^{\prime}}(\mathbf{x}) \quad \text { on } \Gamma_{C}^{k}
$$

The equation is solved only approximately, by interpolating at a set of suitable points. Therefore, the related minimal extension operator is called collocation-based extension operator (CEO). This will be discussed in more detail in Section 3.4.1.

Recall that $M_{h}^{k} u_{h}^{k^{\prime}}=u_{M h}^{k}$, which we can write as a sum $u_{M h}^{k}=u_{M c h}^{k}+u_{M \Phi h}^{k}$, where $u_{M c h}^{k} \in V_{c h}^{k}$ and $u_{M \Phi h}^{k} \in V_{\Phi h}^{k}$. Since $u_{M \Phi h}^{k}$ vanishes at $\Gamma_{C}^{k}$, only the non-vanishing term $u_{M c h}^{k}$ is determined by equation (22). Hence, when considering CEO, the operator is already determined completely by the interpolation.

If $\operatorname{Im}\left(M_{h}^{k}\right) \supset V_{c h}^{k}$, then the function $u_{M h}^{k}=M_{h}^{k} u_{h}^{k^{\prime}}$ is obtained by solving (22) and additionally discretizing the harmonic equation on the parameter domain, i.e.,

$$
\Delta_{\boldsymbol{\xi}}\left[u_{M h}^{k} \circ \mathbf{G}^{k}\right](\boldsymbol{\xi}) \approx 0 \quad \text { in }\left(\mathbf{G}^{k}\right)^{-1}\left(\Phi^{12}\right) \subset \hat{\Omega^{k}}
$$

where $\Delta_{\xi}$ is the Laplace operator on the parametric domain. We call this a quasi-harmonic extension operator (QEO). The details of this approach will be explained in Section 3.4.2.

\subsubsection{Discretization by collocation}

In the following we describe how to solve equation (22) by collocation. Let us recall that

$$
u_{M h}^{k}(\mathbf{x})=\sum_{i \in \mathcal{I}_{\Phi c}^{k}} d_{i}^{k} \beta_{i}^{k}(\mathbf{x})
$$

so we obtain

$$
u_{M h}^{k}(\mathbf{x})=\sum_{i \in \mathcal{I}_{\Phi c}^{k}} d_{i}^{k} \beta_{i}^{k}(\mathbf{x}) \approx \sum_{i \in \mathcal{I}_{0}^{k^{\prime}}} c_{i}^{k^{\prime}} \beta_{i}^{k^{\prime}}(\mathbf{x})+\sum_{i \in \mathcal{I}_{\Phi c}^{k^{\prime}}} d_{i}^{k^{\prime}} \beta_{i}^{k^{\prime}}(\mathbf{x})=u_{h}^{k^{\prime}}(\mathbf{x}) \quad \text { on } \Gamma_{C}^{k} .
$$

Since $\beta_{i}^{k}$ vanish at $\Gamma_{C}^{k}$ for $i \in \mathcal{I}_{\Phi}^{k}$, this reduces to

$$
\sum_{i \in \mathcal{I}_{c}^{k}} d_{i}^{k} \beta_{i}^{k}(\mathbf{x}) \approx \sum_{i \in \mathcal{I}_{0}^{k^{\prime}}} c_{i}^{k^{\prime}} \beta_{i}^{k^{\prime}}(\mathbf{x})+\sum_{i \in \mathcal{I}_{c}^{k^{\prime}}} d_{i}^{k^{\prime}} \beta_{i}^{k^{\prime}}(\mathbf{x}) \quad \text { on } \Gamma_{C}^{k}
$$


To determine the coefficients $d_{i}^{k}$ for $i \in \mathcal{I}_{c}^{k}$ we define a set of suitable collocation points $\left(\mathbf{x}_{\ell}^{k}\right)_{\ell \in \mathcal{I}_{c}^{k}}$, with $\mathbf{x}_{\ell}^{k} \in \Gamma_{C}^{1}$, and obtain the set of equations

$$
\sum_{i \in \mathcal{I}_{c}^{k}} d_{i}^{k} \beta_{i}^{k}\left(\mathbf{x}_{\ell}^{k}\right)=\sum_{i \in \mathcal{I}_{0}^{k^{\prime}}} c_{i}^{k^{\prime}} \beta_{i}^{k^{\prime}}\left(\mathbf{x}_{\ell}^{k}\right)+\sum_{i \in \mathcal{I}_{c}^{k^{\prime}}} d_{i}^{k^{\prime}} \beta_{i}^{k^{\prime}}\left(\mathbf{x}_{\ell}^{k}\right) \quad \text { for all } \ell \in \mathcal{I}_{c}^{k} .
$$

As collocation points $\mathbf{x}_{\ell}^{k}$ one can use the images of the Greville points of the functions $\hat{\beta}_{\ell}^{k}$ under the geometry mapping $\mathbf{G}^{k}$.

Equation (24) can now be expressed in matrix form as follows,

$$
C_{c}^{k} d_{c}^{k}=C_{0}^{k^{\prime}} c_{0}^{k^{\prime}}+C_{d}^{k^{\prime}} d_{c}^{k^{\prime}}
$$

where the matrices $C_{c}^{k}, C_{0}^{k^{\prime}}$ and $C_{d}^{k^{\prime}}$ are given as

$$
\begin{aligned}
\left(C_{c}^{k}\right)_{\ell i}=\beta_{i}^{k}\left(\mathbf{x}_{\ell}^{k}\right), & \ell \in \mathcal{I}_{c}^{k}, i \in \mathcal{I}_{c}^{k}, \\
\left(C_{0}^{k^{\prime}}\right)_{\ell i}=\beta_{i}^{k^{\prime}}\left(\mathbf{x}_{\ell}^{k}\right), & \ell \in \mathcal{I}_{c}^{k}, i \in \mathcal{I}_{0}^{k^{\prime}}, \\
\left(C_{d}^{k^{\prime}}\right)_{\ell i}=\beta_{i}^{k^{\prime}}\left(\mathbf{x}_{\ell}^{k}\right), & \ell \in \mathcal{I}_{c}^{k}, i \in \mathcal{I}_{c}^{k^{\prime}} .
\end{aligned}
$$

3.4.2. Discretization of the quasi-harmonic extension operator (QEO)

In the following we solve (23) with

$$
u_{M h}^{k}(\mathbf{x})=\sum_{i \in \mathcal{I}_{c}^{k}} d_{i}^{k} \beta_{i}^{k}(\mathbf{x})+\sum_{i \in \mathcal{I}_{\Phi}^{k}} d_{i}^{k} \beta_{i}^{k}(\mathbf{x}),
$$

where we assume that the coefficients $d_{i}^{k}$, for $i \in \mathcal{I}_{c}^{k}$, in the first sum are completely determined by the equations (25).

To determine the coefficients $d_{i}^{k}$, for $i \in \mathcal{I}_{\Phi}^{k}$, we follow the approach presented in [42], by applying a finite difference discretization to equation (23) to get a system of linear equations

$$
-d_{i}^{k}+\sum_{j \in \mathcal{N}(i)} \frac{d_{j}^{k}}{|\mathcal{N}(i)|}=0 \quad \text { for } i \in \mathcal{I}_{\Phi}^{k} .
$$

For $i \in \mathcal{I}_{\Phi}^{k}$ the set $\mathcal{N}(i) \subset \mathcal{I}^{k}$ is the set of neighboring indices of $i$. In 1D these are $i-1$ and $i+1$. In 2D (and similarly in higher dimensions) the neighboring indices of $i$ corresponding to the pair $\left(i_{1}, i_{2}\right)$ are given as $\left(i_{1}-1, i_{2}\right),\left(i_{1}+1, i_{2}\right),\left(i_{1}, i_{2}-1\right)$ and $\left(i_{1}, i_{2}+1\right)$, as depicted in Figure 3.

We moreover assume $d_{j}^{k}=0$ for $j \in \mathcal{N}(i) \backslash \mathcal{I}_{\Phi c}^{k}$, then equation (26) is equivalent to

$$
-d_{i}^{k}+\sum_{j \in \mathcal{N}(i) \cap \mathcal{I}_{\Phi c}^{k}} \frac{d_{j}^{k}}{2^{d}}=0 \quad \text { for } i \in \mathcal{I}_{\Phi}^{k},
$$

which can be written in matrix form as

$$
D_{c}^{k} d_{c}^{1}-D_{\Phi}^{k} d_{\Phi}^{k}=0
$$






Figure 3: Visualization of the neighborhood $\mathcal{N}(i)$ for $i \sim\left(i_{1}, i_{2}\right)$.

Remark 5. For non-uniform meshes one has to adapt equations (26) and (27) accordingly. Alternatively, one may use the uniform finite difference formula (27) as an approximation for quasi-uniform meshes.

Remark 6. It should be noted that the sufficient conditions (7) are not always satisfied for the discrete operators $M_{h}^{k}$, i.e., $\left\|M_{h}^{k} M_{h}^{k^{\prime}}\right\| \neq 0$. However, the operators are constructed in such a way that as $h \rightarrow 0$, the violation becomes less severe. Hence the contraction conditions (6) are satisfied for sufficiently small $h$.

\subsection{Final system}

After combining and properly rearranging the equations (21), (25) and (28), we obtain the following global system

$$
\underbrace{\left(\begin{array}{cc|cccc}
K_{0}^{1} & 0 & K_{c}^{1} & 0 & K_{\Phi}^{1} & 0 \\
0 & K_{0}^{2} & 0 & K_{c}^{2} & 0 & K_{\Phi}^{2} \\
\hline 0 & C_{0}^{2} & -C_{c}^{1} & C_{d}^{2} & 0 & 0 \\
C_{0}^{1} & 0 & C_{d}^{1} & -C_{c}^{2} & 0 & 0 \\
0 & 0 & D_{c}^{1} & 0 & -D_{\Phi}^{1} & 0 \\
0 & 0 & 0 & D_{c}^{2} & 0 & -D_{\Phi}^{2}
\end{array}\right)}_{=: K_{g}}\left(\begin{array}{c}
\mathbf{c}_{0}^{1} \\
\mathbf{c}_{0}^{2} \\
\hline \mathbf{d}_{c}^{1} \\
\mathbf{d}_{c}^{2} \\
\mathbf{d}_{\Phi}^{1} \\
\mathbf{d}_{\Phi}^{2}
\end{array}\right)=\left(\begin{array}{c}
\mathbf{f}^{1} \\
\mathbf{f}^{2} \\
\hline 0 \\
0 \\
0 \\
0
\end{array}\right) .
$$

The approximation of the exact solution $u$ of Problem 1 restricted to a patch $\Omega^{k}$ is then given by

$$
\left.u\right|_{\Omega^{k}} \approx u_{0 h}^{k}+u_{M h}^{k}=\sum_{i \in \mathcal{I}_{0}^{k} \backslash \mathcal{I}_{\Phi}^{k}} c_{i}^{k} \beta_{i}^{k}(\mathbf{x})+\sum_{i \in \mathcal{I}_{\Phi}^{k}}\left(c_{i}^{k}+d_{i}^{k}\right) \beta_{i}^{k}(\mathbf{x})+\sum_{i \in \mathcal{I}_{c}^{k}} d_{i}^{k} \beta_{i}^{k}(\mathbf{x}) .
$$


Note that, when using CEO, the coefficient vectors $\mathbf{d}_{\Phi}^{1}, \mathbf{d}_{\Phi}^{2}$ are omitted and the final system is given by the first (top-left) four-by-four blocks only:

$$
\left(\begin{array}{cc|cc}
K_{0}^{1} & 0 & K_{c}^{1} & 0 \\
0 & K_{0}^{2} & 0 & K_{c}^{2} \\
\hline 0 & C_{0}^{2} & -C_{c}^{1} & C_{d}^{2} \\
C_{0}^{1} & 0 & C_{d}^{1} & -C_{c}^{2}
\end{array}\right)\left(\begin{array}{c}
\mathbf{c}_{0}^{1} \\
\mathbf{c}_{0}^{2} \\
\hline \mathbf{d}_{c}^{1} \\
\mathbf{d}_{c}^{2}
\end{array}\right)=\left(\begin{array}{c}
\mathbf{f}^{1} \\
\mathbf{f}^{2} \\
\hline 0 \\
0
\end{array}\right)
$$

In this case the approximate solution is given by

$$
\left.u\right|_{\Omega^{k}} \approx u_{0 h}^{k}+u_{M h}^{k}=\sum_{i \in \mathcal{I}_{0}^{k} \backslash \mathcal{I}_{\Phi}^{k}} c_{i}^{k} \beta_{i}^{k}(\mathbf{x})+\sum_{i \in \mathcal{I}_{\Phi}^{k}} c_{i}^{k} \beta_{i}^{k}(\mathbf{x})+\sum_{i \in \mathcal{I}_{c}^{k}} d_{i}^{k} \beta_{i}^{k}(\mathbf{x}) .
$$

Theorem 2. If $\left(\mathbf{c}_{0}^{1}, \mathbf{c}_{0}^{2}, \mathbf{d}_{c}^{1}, \mathbf{d}_{c}^{2}, \mathbf{d}_{\Phi}^{1}, \mathbf{d}_{\Phi}^{2}\right)^{T}$ is a solution of $(29)$, then $\left(\tilde{\mathbf{c}}_{0}^{1}, \tilde{\mathbf{c}}_{0}^{2}, \mathbf{d}_{c}^{1}, \mathbf{d}_{c}^{2}\right)^{T}$ is a solution of (31), where $\tilde{\mathbf{c}}_{0}^{k}=\left(\tilde{\mathbf{c}}_{i}^{k}\right)_{i \in \mathcal{I}_{0}^{k}}$, with

$$
\tilde{\mathbf{c}}_{i}^{k}= \begin{cases}c_{i}^{k} & \text { for } i \in \mathcal{I}_{0}^{k} \backslash \mathcal{I}_{\Phi}^{k} \\ c_{i}^{k}+d_{i}^{k} & \text { for } i \in \mathcal{I}_{\Phi}^{k} .\end{cases}
$$

Proof. It is easy to see that the first two equations in (31) are satisfied by definition. The last two equations are satisfied, since $C_{0}^{k} \tilde{c}_{0}^{k}=C_{0}^{k} c_{0}^{k}$. This is due to the fact that the rows in the matrix $C_{0}^{k}$ are zero for all row indices $i \in \mathcal{I}_{\Phi}^{k}$ where the vectors $\tilde{\mathbf{c}}_{0}^{k}$ and $\mathbf{c}_{0}^{k}$ differ, i.e., $\left(C_{0}^{k}\right)_{\ell i}=\beta_{i}^{k}\left(\mathbf{x}_{\ell}^{k^{\prime}}\right)=0$, for $\mathbf{x}_{\ell}^{k^{\prime}} \in \Gamma_{C}^{k^{\prime}}$ and $i \in \mathcal{I}_{\Phi}^{k}$.

It means, if Problem 4 has a unique solution with respect to QEO, it also has a solution with respect to CEO. However, the theorem does not guarantee the uniqueness of the solution with respect to $\mathrm{CEO}$.

\section{Existence and uniqueness for the discretized problem}

The continuous Problem 2 always has a unique solution, thanks to Theorem 1, where the equivalence of solutions of Problems 1 and 2 was shown. However, Theorem 1 does not apply to Problem 4. Instead we will show by numerical experiments the existence and uniqueness of the solution. Therefore, we need to analyze the regularity of the system matrix $K_{g}$. However, we do not analyze the full matrix directly but make a Schur complement reformulation.

We write the final matrix as presented in (29) as a block matrix

$$
K_{g}=\left(\begin{array}{cc}
A & B \\
C & D
\end{array}\right)
$$


where

$$
\begin{aligned}
& A=\left(\begin{array}{cc}
K_{0}^{1} & 0 \\
0 & K_{0}^{2}
\end{array}\right), \quad B=\left(\begin{array}{cccc}
K_{c}^{1} & 0 & K_{\mathcal{E}}^{1} & 0 \\
0 & K_{c}^{2} & 0 & K_{\mathcal{E}}^{2}
\end{array}\right), \\
& C=\left(\begin{array}{cc}
0 & C_{0}^{2} \\
C_{0}^{1} & 0 \\
0 & 0 \\
0 & 0
\end{array}\right), \quad D=\left(\begin{array}{cccc}
-C_{c}^{1} & C_{d}^{2} & 0 & 0 \\
C_{d}^{1} & -C_{c}^{2} & 0 & 0 \\
D_{c}^{1} & 0 & -D_{\Phi}^{1} & 0 \\
0 & D_{c}^{2} & 0 & -D_{\Phi}^{2}
\end{array}\right) \text {. }
\end{aligned}
$$

It can be checked easily that the block matrix $D$ is invertible. This is due to the interpolation together with the finite difference scheme having a unique solution. Then the Schur complement matrix with respect to the block $D$ of the matrix $K_{g}$ is obtained by

$$
\tilde{K}_{g}:=A-B D^{-1} C .
$$

Remark 7. We recall the descretized continuity condition

$$
u_{M h}^{k}-M_{h}^{k}\left(u_{0 h}^{k^{\prime}}+u_{M h}^{k^{\prime}}\right)=0 \quad \text { on } \Omega^{k}, \quad k \in\{1,2\},
$$

which we can write in block operator form as

$$
\left(\begin{array}{cc|cc}
0 & M_{h}^{1} & -I_{h}^{1} & M_{h}^{1} \\
M_{h}^{2} & 0 & M_{h}^{2} & -I_{h}^{2}
\end{array}\right)\left(\begin{array}{c}
u_{0 h}^{1} \\
u_{0 h}^{2} \\
u_{M h}^{1} \\
u_{M h}^{2}
\end{array}\right)=\left(\begin{array}{l}
0 \\
0
\end{array}\right)
$$

Writing (37) in terms of the coefficients $\mathbf{c}_{0}^{k}, \mathbf{d}_{c}^{k}$ and $\mathbf{d}_{\Phi}^{k}$ we obtain, after some calculations, the block matrix equation

$$
C\left(\begin{array}{c}
\mathbf{c}_{0}^{1} \\
\mathbf{c}_{0}^{2}
\end{array}\right)+D\left(\begin{array}{c}
\mathbf{d}_{c}^{1} \\
\mathbf{d}_{c}^{2} \\
\mathbf{d}_{\Phi}^{1} \\
\mathbf{d}_{\Phi}^{2}
\end{array}\right)=\left(\begin{array}{l}
0 \\
0 \\
0 \\
0
\end{array}\right)
$$

where $C$ and $D$ are the block matrices as defined in (34). Hence, we obtain

$$
(-D)^{-1} C\left(\begin{array}{c}
\mathbf{c}_{0}^{1} \\
\mathbf{c}_{0}^{2}
\end{array}\right)=\left(\begin{array}{c}
\mathbf{d}_{c}^{1} \\
\mathbf{d}_{c}^{2} \\
\mathbf{d}_{\Phi}^{1} \\
\mathbf{d}_{\Phi}^{2}
\end{array}\right)
$$


which is again equivalent to

$$
\left(\begin{array}{cc}
I_{h}^{1} & -M_{h}^{1} \\
-M_{h}^{2} & I_{h}^{2}
\end{array}\right)^{-1}\left(\begin{array}{cc}
0 & M_{h}^{1} \\
M_{h}^{2} & 0
\end{array}\right)\left(\begin{array}{l}
u_{0 h}^{1} \\
u_{0 h}^{2}
\end{array}\right)=\left(\begin{array}{l}
u_{M h}^{1} \\
u_{M h}^{2}
\end{array}\right)
$$

From this we conclude

$$
\underbrace{\left[\left(\begin{array}{cc}
I_{h}^{1} & 0 \\
0 & I_{h}^{2}
\end{array}\right)+\left(\begin{array}{cc}
I_{h}^{1} & -M_{h}^{1} \\
-M_{h}^{2} & I_{h}^{2}
\end{array}\right)^{-1}\left(\begin{array}{cc}
0 & M_{h}^{1} \\
M_{h}^{2} & 0
\end{array}\right)\right]}_{\sum_{\ell=0}^{\infty}\left(\begin{array}{cc}
0 & M_{h}^{1} \\
M_{h}^{2} & 0
\end{array}\right)^{\ell}}\left(\begin{array}{l}
u_{0 h}^{1} \\
u_{0 h}^{2}
\end{array}\right)=\left(\begin{array}{l}
u_{h}^{1} \\
u_{h}^{2}
\end{array}\right) .
$$

Hence, solving the system obtained by the Schur complement matrix $\tilde{K}_{g}$ can be seen as a discretization of Problem 2.

What remains to be shown is whether or not the matrix $\tilde{K}_{g}$ is invertible. Let

$$
\widetilde{\mathcal{A}}\left(\left(u_{0 h}^{1}, u_{0 h}^{2}\right),\left(v_{0 h}^{1}, v_{0 h}^{2}\right)\right)=\mathbf{v}^{T} \tilde{K}_{g} \mathbf{u}
$$

be the induced bilinear form, with $\mathbf{u}$ and $\mathbf{v}$ being the coefficient vectors of $\left(u_{0 h}^{1}, u_{0 h}^{2}\right)$ and $\left(v_{0 h}^{1}, v_{0 h}^{2}\right)$, respectively. To study the solvability, we will show numerically that the bilinear form $\widetilde{\mathcal{A}}$ is bounded and coercive, i.e.,

$$
\widetilde{\mathcal{A}}\left(u_{h}, v_{h}\right) \leq \bar{\mu}\left\|u_{h}\right\|_{H^{1}}\left\|v_{h}\right\|_{H^{1}}
$$

and

$$
\widetilde{\mathcal{A}}\left(u_{h}, u_{h}\right) \geq \underline{\mu}\left\|u_{h}\right\|_{H^{1}}^{2},
$$

where $u_{h}=\left(u_{0 h}^{1}, u_{0 h}^{2}\right), v_{h}=\left(v_{0 h}^{1}, v_{0 h}^{2}\right)$ and $\left\|u_{h}\right\|_{H^{1}}=\left\|\left(u_{0 h}^{1}, u_{0 h}^{2}\right)\right\|_{H^{1}\left(\Omega^{1}\right) \times H^{1}\left(\Omega^{2}\right)}$.

Let $K_{g}^{s}=\frac{1}{2}\left(\tilde{K}_{g}+\tilde{K}_{g}^{T}\right)$ and $N$ be the matrix related to the $H^{1}$-norm, i.e., $\mathbf{u}^{T} N \mathbf{u}=\left\|u_{h}\right\|_{H^{1}}^{2}$. Computing the extremal eigenvalues of the matrix $K_{g}^{s} N^{-1}$

$$
\lambda_{\min } \leq \frac{\mathbf{u}^{T} K_{g}^{s} \mathbf{u}}{\mathbf{u}^{T} N \mathbf{u}} \leq \lambda_{\max }
$$

we get that

$$
\lambda_{\min }\left\|u_{h}\right\|_{H^{1}}^{2}=\lambda_{\min } \mathbf{u}^{T} N \mathbf{u} \leq \mathbf{u}^{T} K_{g}^{s} \mathbf{u}=\mathbf{u}^{T} \tilde{K}_{g} \mathbf{u}=\widetilde{\mathcal{A}}\left(u_{h}, u_{h}\right) \leq \lambda_{\max }\left\|u_{h}\right\|_{H^{1}}^{2} .
$$

Hence, having a positive minimal eigenvalue $\lambda_{\min }$ guarantees that the bilinear form $\alpha$ is coercive, therefore the matrix $\tilde{K}_{g}$ is invertible and so is the original system matrix $K_{g}$. Moreover, the conditioning of the matrix $K_{g}^{s} N^{-1}$ depends on the ratio $\lambda_{\max } / \lambda_{\min }$. 




(a) Degenerate overlap

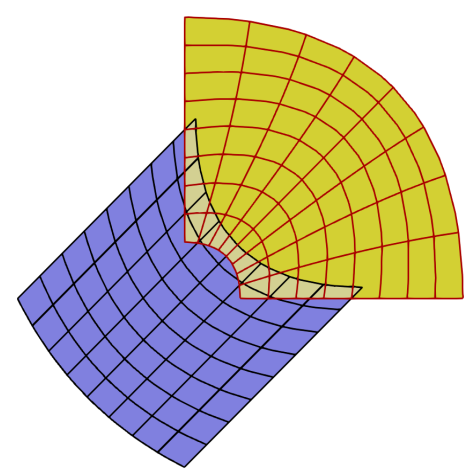

(b) Small overlap

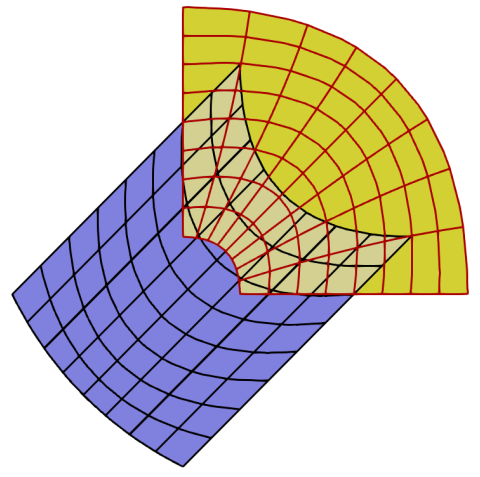

(c) Large overlap

Figure 4: Two-patch setting for different size of overlaps.

We consider three parameterizations of the same domain as illustrated in Figure 4, where a degenerate overlap appears when the boundaries of two overlapping subdomains touch each other in one point within the overlap region. While the domain itself cannot be parameterized by a single regular spline patch, it can be represented as the union of two patches with overlap. For these examples we compute $\lambda_{\min }$ and $\lambda_{\max }$ for varying degree and refinement level.

In Figures 5(a) and 5(b) we plot the computed eigenvalues using CEO and QEO, respectively. We compare the results for different degrees (left) and different overlap sizes (right). As we can see in Figure 5(a), both minimal and maximal eigenvalues obtained using CEO are diverging for all tested examples as the mesh size tends to zero. In comparison, the spectral behavior is improved significantly when using QEO, as can be observed in Figure 5(b).

For the domain with a large overlap, the minimal eigenvalues are bounded away from zero and the maximal eigenvalues are increasing only moderately for all tested degrees. Hence, we assume that sufficiently large overlap sizes guarantee that Problem 4 has a unique solution when using QEO and that the system matrix is well-conditioned.

A similar behavior is observed for the example with small overlap, where the ratio of eigenvalues is increasing at first but decreasing again after some refinement steps.

In case of the degenerate overlap, the minimal eigenvalue becomes negative at some point. Nonetheless, the spectrum using QEO is smaller than when using CEO.

When increasing the degree of the basis functions, the value of the minimal (maximal) eigenvalue decreases (increases). We suspect that this is due to the increase of the support size of the basis functions.

Summing up, using QEO we need enough basis functions in the overlap region to obtain coercivity and stability which can be guaranteed by $h$-refinement. Hence we can conclude that Problem 4 
Large overlap

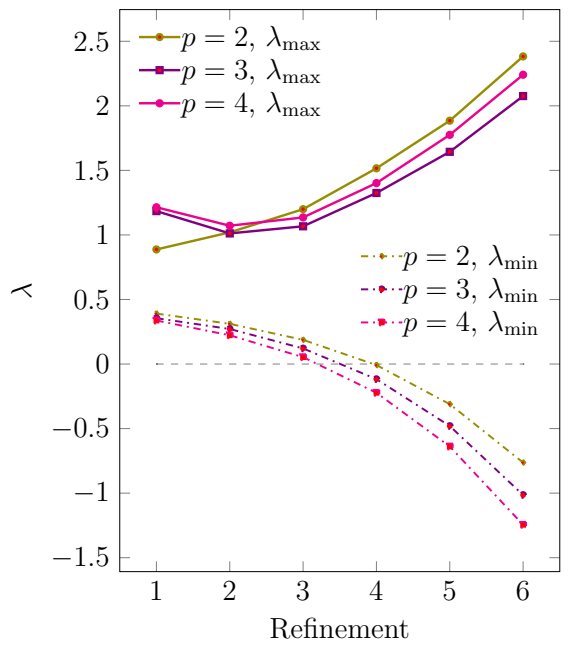

$p=2$

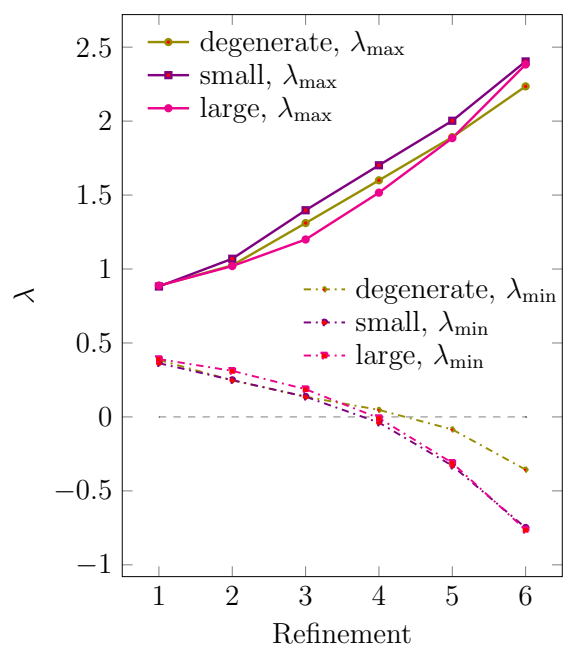

(a) Spectrum of $\tilde{K}_{g}$ with respect to CEO.


(b) Spectrum of $\tilde{K}_{g}$ with respect to QEO.

Figure 5: Minimal and maximal eigenvalues for different degree (left) and different overlap size (right) for the domains in Figure 4, using CEO (top) and QEO (bottom).

has a unique solution in that case. According to Theorem 2, it also has a solution using CEO. It is worth to mention here, that, while the theorem does not guarantee uniqueness, we always found 
that the CEO based formulation is uniquely solvable and stable.

\section{Numerical experiments}

We study the OMP method for some numerical examples. According to Theorem 2, any solution using QEO directly yields a solution using CEO. After presenting a comparison between CEO and QEO with respect to computation times and convergence order (Sections 5.1 and 5.2, respectively), we only use CEO for all following examples. In Section 5.3 we solve the Poisson problem with non-homogeneous boundary condition and given right-hand side on different domains, which are a union of two or more subdomains. In Section 5.4 we compare the OMP method with two versions of the Schwarz method. We implemented this work using the $\mathrm{C}++$ library $\mathbf{G}+$ Smo [43].

\subsection{Time comparison between $C E O$ and $Q E O$}

We compare the performance of the OMP method for CEO and QEO with respect to the computation time. For QEO the supports of the additional basis functions need to be entirely in the overlap region. Hence, to obtain a difference between CEO and QEO, a domain with sufficiently large overlap is required. Otherwise, the final systems related to CEO and QEO are of similar size and the computation time regarding the extension operators is not considerably different. We solve the Poisson problem on the domain depicted in Figure 4(c) with respect to CEO and QEO having the exact solution

$$
u(x, y)=\sin (x+y)
$$

Table 1 and Figure 6 indicate results for degree two and different levels of refinement. Moreover, in Table 2 we compare the two extension operators for varying degree on a fixed refinement level. The observed results show that the OMP method with respect to CEO is almost two times faster than QEO.

The results also demonstrate experimentally that the computation times for both methods have the same order of magnitude. Consequently, the observations regarding CEO, which are represented in the following subsections are expected to carry over to QEO as well.

\subsection{Convergence comparison between $C E O$ and $Q E O$}

We compare the performance of the OMP method concerning CEO and QEO with respect to the $H^{1}$ and $L^{2}$ errors. Therefore, we consider the Poisson problem on domain 4(c) and the exact

solution (45). The results presented in Table 3 are confirming Theorem 2 . We conjecture that the obtained errors are the same and that the observed differences are due to the iterative BiCG solver. 


\begin{tabular}{|r||r|r|r||r|r|r||r|}
\hline \multicolumn{1}{|c||}{} & \multicolumn{3}{c||}{ CEO } & \multicolumn{3}{c||}{ QEO } & time ratio \\
\hline ref & time & \#it & size & time & \#it & size & QEO/CEO \\
\hline 1 & $9.9 \times 10^{-4}$ & 31 & 648 & $2.2 \times 10^{-3}$ & 37 & 746 & 2.22 \\
2 & $7.5 \times 10^{-3}$ & 63 & 2312 & $1.3 \times 10^{-2}$ & 74 & 2834 & 1.73 \\
3 & $6 \times 10^{-2}$ & 128 & 8712 & $1 \times 10^{-1}$ & 162 & 11136 & 1.66 \\
4 & $5.5 \times 10^{-1}$ & 234 & 33800 & 1 & 316 & 44186 & 1.81 \\
5 & 4.9 & 499 & 133128 & 8.8 & 649 & 176006 & 1.79 \\
6 & 44 & 949 & 528392 & 86 & 1340 & 702720 & 1.95 \\
\hline
\end{tabular}

Table 1: Time comparison with respect to refinement levels for CEO and QEO on domain 4(c) and p $=2$. Here and in the following tables \#it stands for solver iterations.

\begin{tabular}{|r||r|r|r||r|r|r||r|}
\hline \multicolumn{1}{|c||}{} & \multicolumn{3}{c||}{ CEO } & \multicolumn{3}{|c||}{ QEO } & time ratio \\
\hline degree & time & \#it & size & time & \#it & size & QEO/CEO \\
\hline 2 & 44 & 949 & 528392 & 86 & 1340 & 702720 & 1.95 \\
3 & 68.8 & 831 & 530450 & 131 & 1159 & 704734 & 1.90 \\
4 & 104 & 756 & 532512 & 201 & 1220 & 706756 & 1.93 \\
\hline
\end{tabular}

Table 2: Time comparison with respect to degree for $\mathrm{CEO}$ and QEO on domain 4(c)

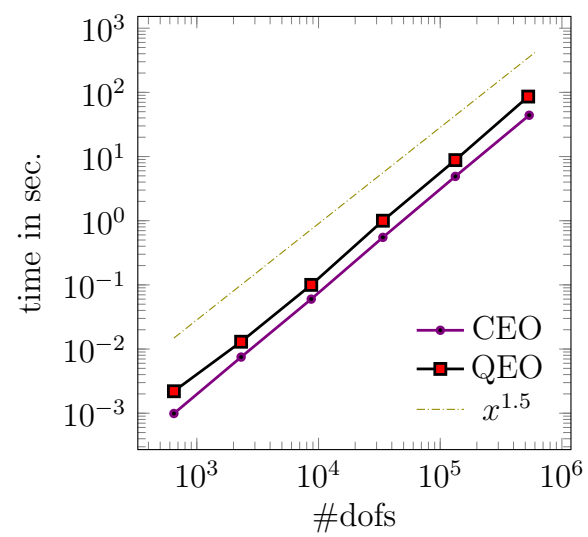

Figure 6: Time comparison with respect to refinement levels for CEO and QEO on domain 4(c).

\subsection{Convergence tests}

In Example 5.3.1, we solve the Poisson problem on domain 4(c), using degree elevation, and for a fixed mesh size. In Example 5.3.2 we consider a half-circle shaped domain, which is the union of two overlapping patches. The two subdomains are sharing a Dirichlet boundary part. A coffee cup shaped domain is considered in Example 5.3.3. The domain is a union of four pairwise overlapping subdomains. In Example 5.3.4 the performance of the OMP method is tested on a $3 D$ domain, which is constructed as the union of two overlapping cubes.

In all three examples we solve the Poisson problem, where the right-hand side $f$ and the Dirichlet 


\begin{tabular}{|r||r|r||r|r||}
\hline \multicolumn{1}{|c||}{} & \multicolumn{2}{c||}{ CEO } & \multicolumn{2}{c||}{ QEO } \\
\hline $\mathrm{h}$ & $L^{2}$-error & $H^{1}$-error & $L^{2}$-error & $H^{1}$-error \\
\hline 1 & 7.42384 & 10.6648 & 7.42384 & 10.6648 \\
0.5 & 3.79419 & 5.51633 & 3.79419 & 5.51633 \\
0.25 & 0.74306 & 1.83793 & 0.74306 & 1.83793 \\
0.125 & 0.0534081 & 0.373055 & 0.0534087 & 0.373055 \\
0.0625 & 0.00463993 & 0.0848575 & 0.00463993 & 0.0848575 \\
0.03125 & 0.000479796 & 0.0206381 & 0.000479798 & 0.0206381 \\
0.015625 & $5.82 \times 10^{-5}$ & 0.00512118 & $5.82 \times 10^{-5}$ & 0.00512118 \\
\hline
\end{tabular}

Table 3: Convergence comparison with respect to the mesh size $h$ and $p=2$ for CEO and QEO on domain 4(c).

boundary conditions are defined by a prescribed exact solution. The numerical solution is computed by solving the global system (31) for varying degree and mesh size. We always compute the $L^{2}$ and $H^{1}$ errors. In all examples the total error is given as the sum of local errors on each subdomain.

\subsubsection{Degree elevation example}

The domain 4(c) is parameterized by biquadratic patches. We consider the exact solution (45) to compute the numerical errors. The results are indicated in Figure 7 for $L^{2}$ and $H^{1}$ errors and for $h=0.0625$.
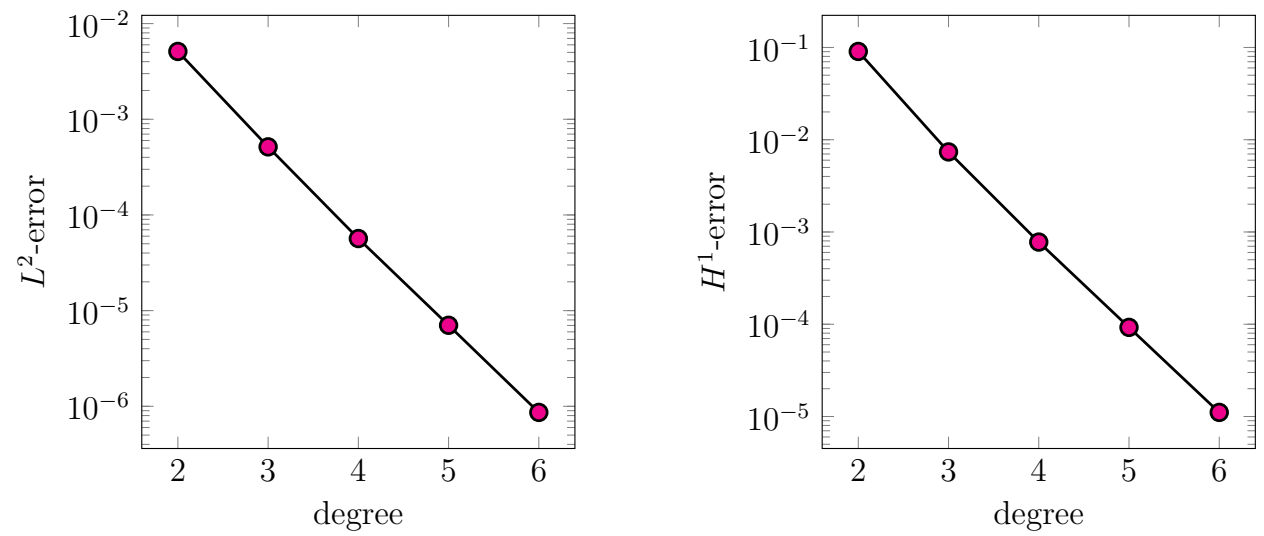

Figure 7: Numerical results related to solving the Poisson problem on domain 4(c) for $h=0.0625$.

\subsubsection{Poisson problem on a half-circle}

The domain illustrated in Figure 8(a) approximates a half-circle with radius 3 around the origin (the domain is parameterized with quadratic B-splines and thus not an exact half-circle). While the domain itself cannot be parameterized by a single regular spline patch, it can be represented as the union of an annulus and a rectangular domain. We consider the exact solution (45). 
The numerical solution with a total of 1512 dofs and using quadratic B-splines, is plotted in Figures $8(\mathrm{~b})$ and $8(\mathrm{c})$. The $L^{2}$ and $H^{1}$ errors are plotted in Figure 8(d). The observed order of convergence is optimal.

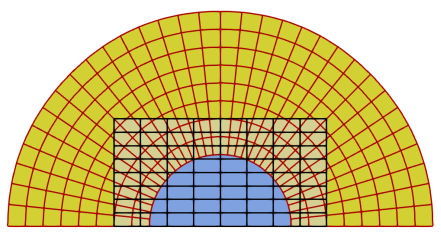

(a) The half-circle domain.


(b) Local numerical solutions.


(c) Both local solutions plotted together.
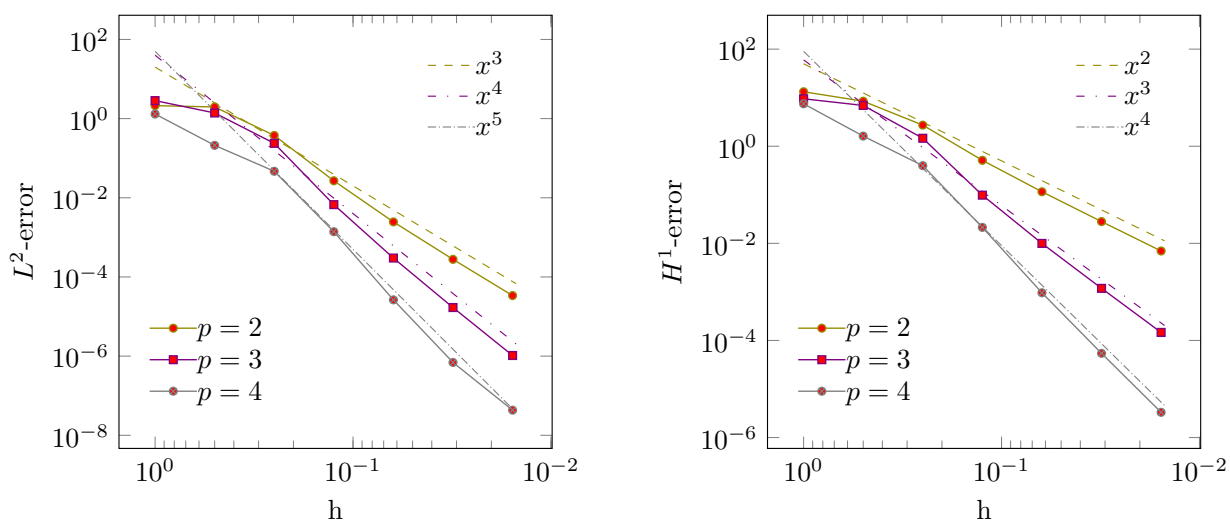

(d) $L^{2}$ (left) and $H^{1}$ (right) errors for the half-circle.

Figure 8: Numerical results related to solving the Poisson problem on the half-circle. 


\subsubsection{Poisson problem on a coffee cup shaped domain}

In this example, we consider the domain depicted in Figure 9(a). The domain is composed of four subdomains parameterized with quadratic B-splines. We consider the following exact solution

$$
u(x, y)=\sin (x) \sin (y)
$$

In Figure 9(c) we plot the numerical solution with a total of 1296 dofs for $p=2$. The convergence of $L^{2}$ and $H^{1}$ errors are shown in Figure $9(\mathrm{~d})$, which verifies the optimal convergence rate for this example.

\subsubsection{Poisson problem on overlapping cubes}

We consider the 3D domain illustrated in Figure 10(a), which is given as the union of two cubes. The exact solution is

$$
u(x, y, z)=\sin (x+y+z) .
$$

The numerical solution for $p=2$ with a total of 2000 dofs is plotted in Figure 10(b).

We again observe the optimal order of convergence for the $L^{2}$ and $H^{1}$ errors in Figure 10(c).

\subsubsection{Linear elasticity problem on a plate with circular hole}

The domain, which is shown in Figure 11(a), is a quarter of a plate with a circular hole. It is represented as a Boolean union of two subdomains, both parameterized by quadratic NURBS. The radius of the hole and the edge length of the quarter are set to one and four, respectively.

On this domain, we consider the same problem as in [44, Section 5.2], where the domain was simply a square.

We recall the exact solution

$$
\begin{aligned}
& u_{1}(x, y)=-\frac{\cos ^{2}(x) \cos (y) \sin (y)}{2} \\
& u_{2}(x, y)=\frac{\cos ^{2}(y) \cos (x) \sin (x)}{2}
\end{aligned}
$$

and right-hand side

$$
\begin{aligned}
& f_{1}(x, y)=\mu \cos (y) \sin (y)\left(1-4 \cos ^{2}(x)\right)-2 x y \cos \left(x^{2} y\right) \\
& f_{2}(x, y)=-\mu \cos (x) \sin (x)\left(1-4 \cos ^{2}(y)\right)-x^{2} \cos \left(x^{2} y\right) .
\end{aligned}
$$

The Lamé parameters are determined by Young's modulus $E=10^{5}$ and Poisson's ratio $\nu=0.3$.

Figure 11(b) visualizes the numerical solution with a total of 2664 dofs (corresponding to $h=$ $0.03125)$ and using quadratic NURBS. The $L^{2}$ and $H^{1}$ errors for various values of the discretization parameter $h$ are plotted in Figure 11(c). The observed order of convergence is optimal. 


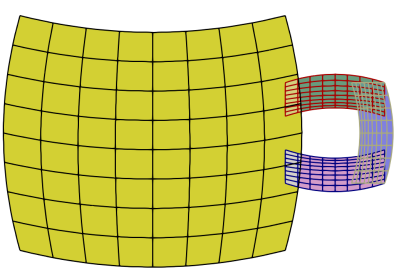

(a) Coffee cup domain.
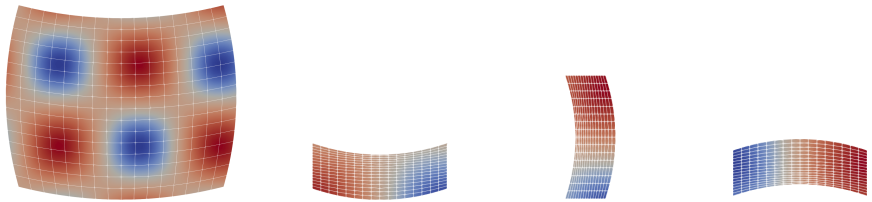

(b) Local numerical solutions.
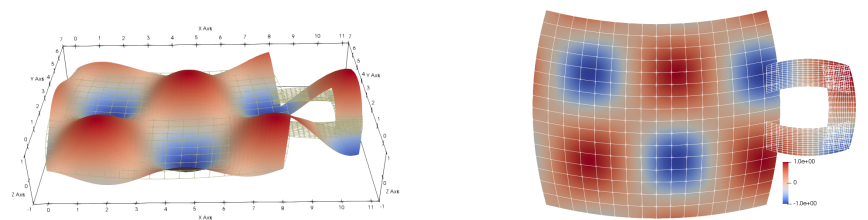

(c) All local solutions plotted together.
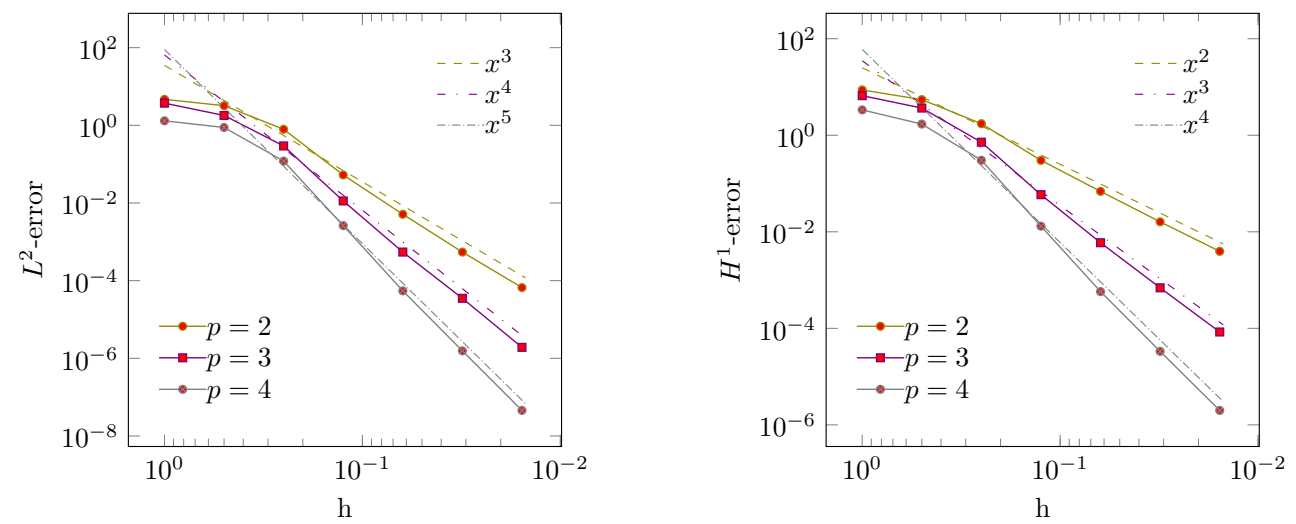

(d) $L^{2}$ (left) and $H^{1}$ (right) errors for the coffee cup shaped domain.

Figure 9: Numerical results related to solving the Poisson problem on the coffee cup shaped domain. 




(a) Overlapping cubes.
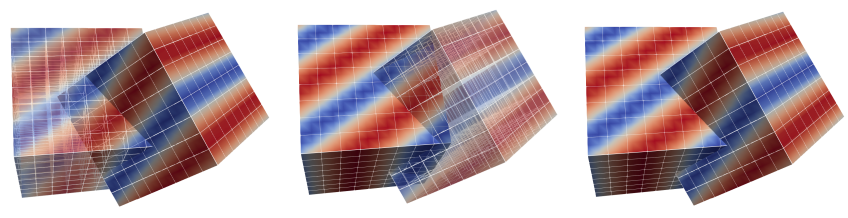

(b) Both local solutions plotted together.
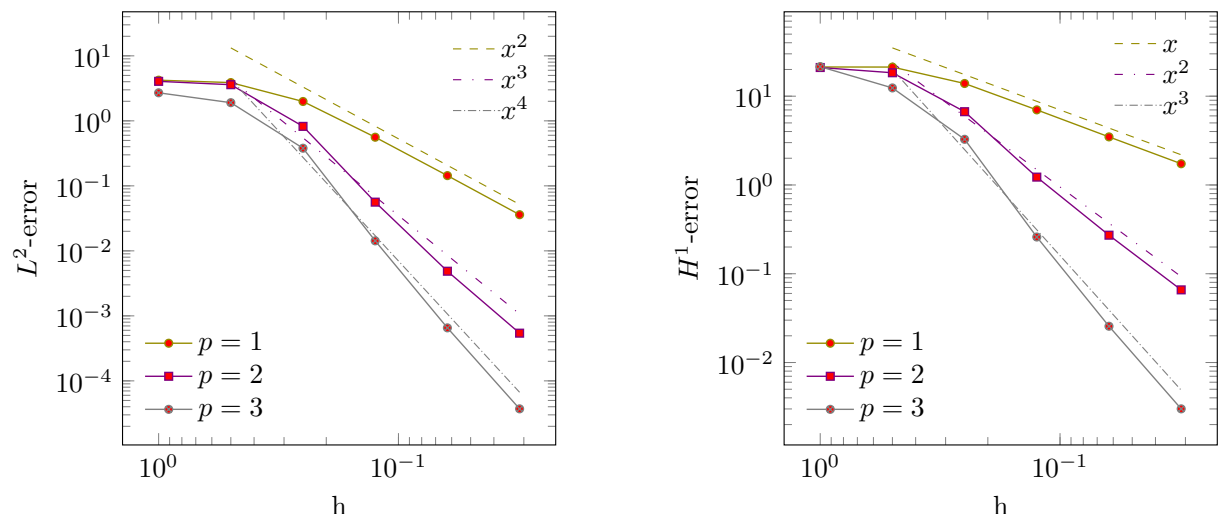

(c) $L^{2}$ (left) and $H^{1}$ (right) errors for the overlapping cubes.

Figure 10: Numerical results related to solving the Poisson problem on the overlapping cubes.

\subsection{Comparison of the OMP method with Schwarz methods}

We compare the OMP method with two different kinds of Schwarz algorithms, additive Schwarz (ADS) and multiplicative Schwarz (MPS).

The ADS and MPS domain decomposition methods, can be used to handle the numerical solution on overlapping subdomains. Moreover, from a linear algebra point of view, the ADS method is a variation of the block Jacobi algorithm and the MPS method corresponds to a symmetric Gauss-Seidel algorithm. In Figure 12 we visualize the dependence of every iterate with respect to the previous solutions. For more information see for instance [45, 46, 47, 48]. In Section 5.4.1 we compare the presented Schwarz algorithms and the proposed OMP method with respect to the computational cost. 


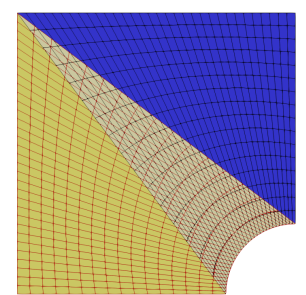

(a) The plate with circular hole.
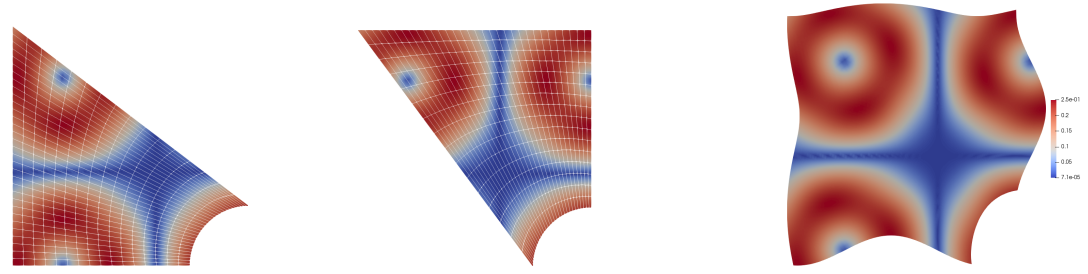

(b) Norm of the displacement on each patch (left and center) and visualization of the deformed plate (right).
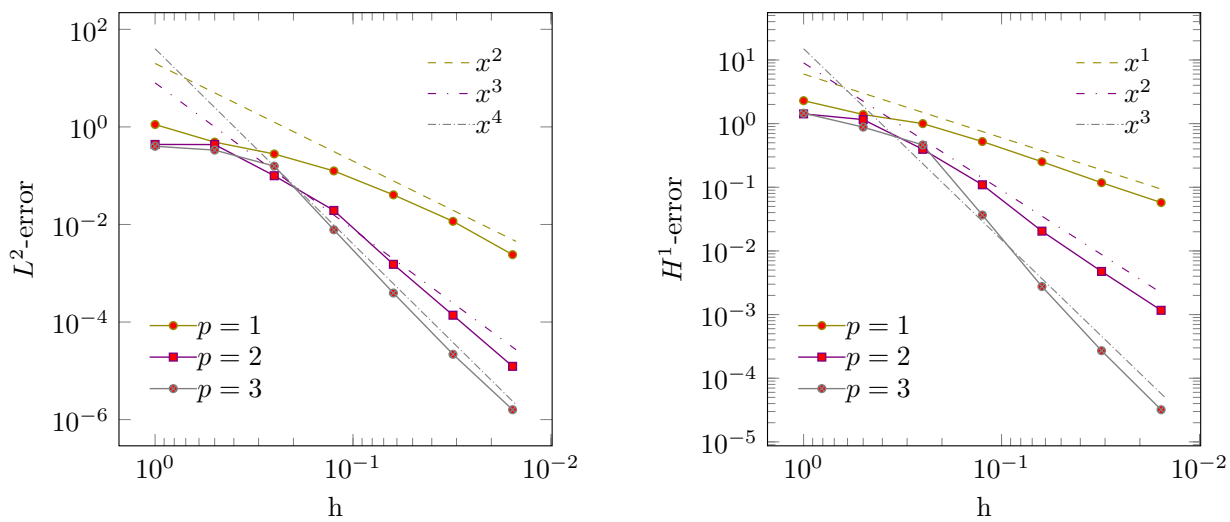

(c) $L^{2}$ (left) and $H^{1}$ (right) errors for the plate with circular hole.

Figure 11: Numerical results related to solving the linear elasticity problem on the plate with circular hole.

In all examples, the Schwarz iteration is terminated if the Euclidean norm of the difference of two successive solutions is below the threshold $10^{-8}$. The maximum number of iterations for the MPS and ADS method is set to 4000.

\subsubsection{Comparison with respect to the computational cost}

We solve the Poisson problem with non-zero Dirichlet boundary condition on a domain, which is composed of two subdomains and with different size of overlaps, as illustrated in Figure 4. Note 

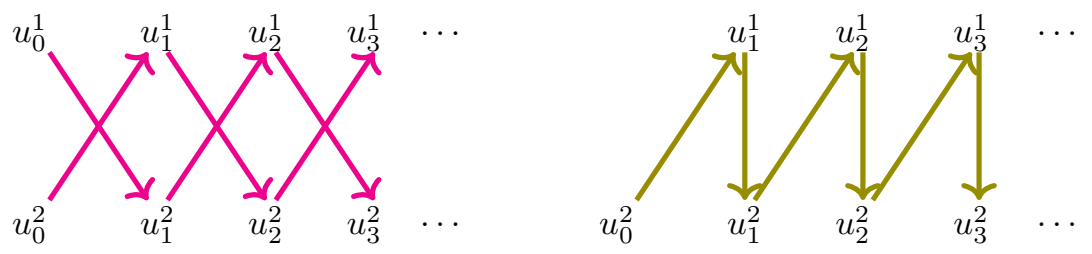

Figure 12: ADS (pink) and MPS (olive) algorithms

that the domain is the same for the three examples and only one subdomain changes. The exact solution

$$
u(x, y)=\sin (\pi x) \sin (\pi y)
$$

is used for all three approaches.

The BiCG solver is employed for solving the systems related to the proposed methods. The tolerance of the solver is set to $10^{-10}$.

The experimental results illustrated in the Tables 4, 5, 6 and Figure 13 for $p=2$. The observed results show that the MPS method is almost two times faster than the ADS method and the OMP method is considerably faster than both Schwarz approaches. Also, the Schwarz approaches are not efficient for degenerate and small overlaps, since we lose convergence after some refinement steps. Also, setting a higher number of maximum iterations of the Schwarz method (> 4000), increases the computation time.

In the presented tables, the listed dofs are related to the MPS and ADS methods, for the OMP method the values have to be multiplied by 2 .
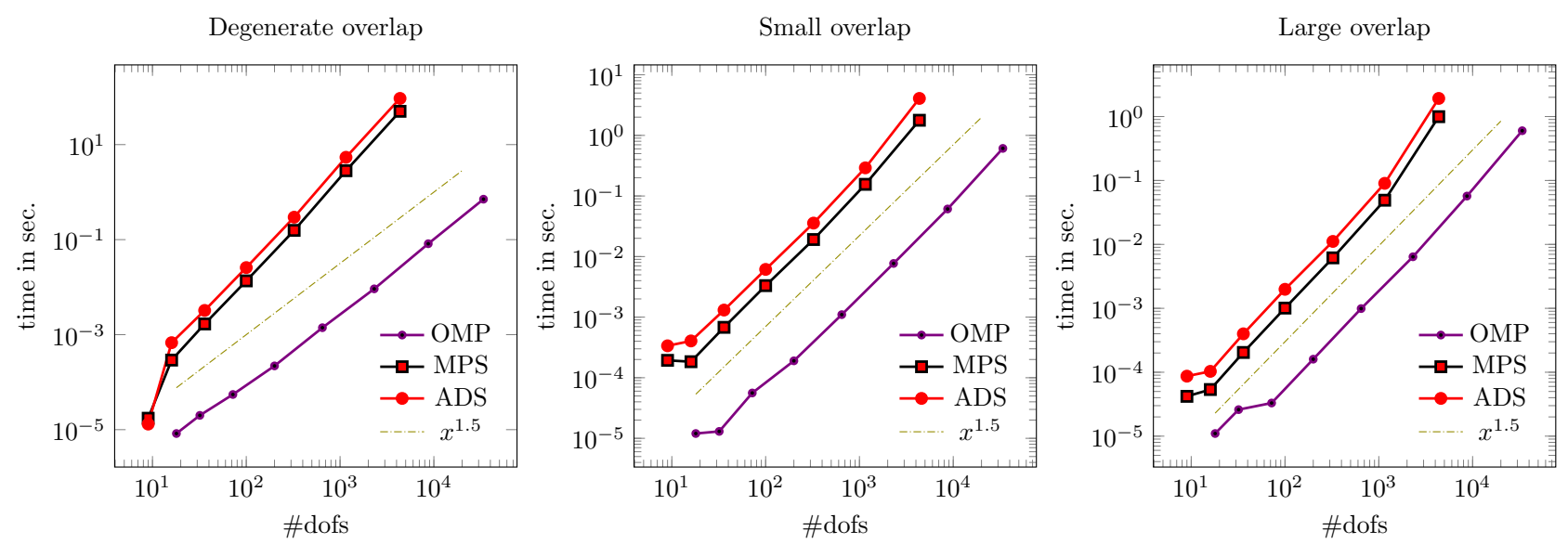

Figure 13: Comparison between the OMP, MPS and ADS methods with respect to the computation time.

As another experiment, we fix the number of dofs, and we elevate the degree of the basis 


\begin{tabular}{|r|r||r|r||r|r|r||r|r|r|}
\hline \multicolumn{9}{|c|}{ Overlap size = Degenerate } \\
\hline \multicolumn{2}{|c||}{$p=2$} & OMP Method & \multicolumn{3}{|c|}{ MPS Method } & \multicolumn{3}{|c|}{ ADS Method } \\
\hline ref & \#dofs & time & \#it & calls & time & \#it & calls & time & \#it \\
\hline 1 & 9 & $8.3 \times 10^{-6}$ & 5 & 2 & $1.7 \times 10^{-5}$ & 18 & 2 & $1.3 \times 10^{-5}$ & 18 \\
2 & 16 & $2 \times 10^{-6}$ & 9 & 48 & $2.9 \times 10^{-4}$ & 490 & 113 & $6.7 \times 10^{-4}$ & 1140 \\
3 & 36 & $5.5 \times 10^{-6}$ & 15 & 83 & $1.6 \times 10^{-3}$ & 1428 & 160 & $3.2 \times 10^{-3}$ & 2738 \\
4 & 100 & $2.2 \times 10^{-4}$ & 26 & 139 & $1.3 \times 10^{-2}$ & 3501 & 268 & $2.5 \times 10^{-2}$ & 6726 \\
5 & 324 & $1.4 \times 10^{-3}$ & 48 & 291 & $1.5 \times 10^{-1}$ & 11103 & 560 & $2.9 \times 10^{-1}$ & 21330 \\
6 & 1156 & $9.2 \times 10^{-3}$ & 83 & 660 & 2.8 & 51435 & 1268 & 5.4 & 98742 \\
7 & 4356 & $8.2 \times 10^{-2}$ & 159 & 1490 & 50.2 & 229183 & 2799 & 93.8 & 430450 \\
8 & 16900 & $7.1 \times 10^{-1}$ & 309 & - & $>10$ min & - & - & $>10 \mathrm{~min}$ & - \\
\hline
\end{tabular}

Table 4: Comparison between the OMP, MPS, and ADS methods. Here and in the following tables "calls" stands for the number of Schwarz iterations.

\begin{tabular}{|r|r||r|r||r|r|r||r|r|r|}
\hline \multicolumn{9}{|c|}{ Overlap size = Small } \\
\hline \multicolumn{2}{|c||}{$p=2$} & \multicolumn{1}{|c|}{ OMP Method } & \multicolumn{3}{|c|}{ MPS Method } & \multicolumn{3}{|c|}{ ADS Method } \\
\hline ref & \#dofs & time & \#it & calls & time & \#it & calls & time & \#it \\
\hline 1 & 9 & $1.2 \times 10^{-5}$ & 13 & 57 & $1.9 \times 10^{-4}$ & 348 & 110 & $3.3 \times 10^{-4}$ & 666 \\
2 & 16 & $1.3 \times 10^{-5}$ & 11 & 30 & $1.8 \times 10^{-4}$ & 310 & 62 & $4.04 \times 10^{-4}$ & 630 \\
3 & 36 & $5.6 \times 10^{-5}$ & 15 & 32 & $6.7 \times 10^{-4}$ & 593 & 62 & $1.3 \times 10^{-3}$ & 1132 \\
4 & 100 & $1.9 \times 10^{-4}$ & 21 & 33 & $003.3 \times 10^{-3}$ & 850 & 64 & $6.1 \times 10^{-3}$ & 1625 \\
5 & 324 & $1.1 \times 10^{-3}$ & 35 & 34 & $1.9 \times 10^{-2}$ & 1341 & 66 & $3.5 \times 10^{-2}$ & 2569 \\
6 & 1156 & $7.7 \times 10^{-3}$ & 65 & 35 & $1.5 \times 10^{-1}$ & 2771 & 68 & $2.9 \times 10^{-1}$ & 5314 \\
7 & 4356 & $6.1 \times 10^{-2}$ & 128 & 52 & 1.7 & 8090 & 121 & 4.07 & 18630 \\
8 & 16900 & $6.1 \times 10^{-1}$ & 243 & - & $>10 \mathrm{~min}$ & - & - & $>10 \mathrm{~min}$ & - \\
\hline
\end{tabular}

Table 5: Comparison between the OMP, MPS, and ADS methods.

\begin{tabular}{|c|c|c|c|c|c|c|c|c|c|}
\hline \multicolumn{10}{|c|}{ Overlap size $=$ Large } \\
\hline \multicolumn{2}{|c|}{$p=2$} & \multicolumn{2}{|c|}{ OMP Method } & \multicolumn{3}{|c|}{ MPS Method } & \multicolumn{3}{|c|}{ ADS Method } \\
\hline ref & \#dofs & time & \#it & calls & time & $\#$ it & calls & time & \#it \\
\hline 1 & 9 & $1.1 \times 10^{-5}$ & 12 & 12 & $4.19 \times 10^{-5}$ & 77 & 24 & $8.67 \times 10^{-5}$ & 148 \\
\hline 2 & 16 & $2.6 \times 10^{-5}$ & 11 & 8 & $5.36 \times 10^{-5}$ & 88 & 17 & $1.03 \times 10^{-4}$ & 177 \\
\hline 3 & 36 & $3.3 \times 10^{-5}$ & 12 & 9 & $2.03 \times 10^{-4}$ & 170 & 19 & $3.98 \times 10^{-4}$ & 339 \\
\hline 4 & 100 & $1.6 \times 10^{-4}$ & 18 & 9 & $10.07 \times 10^{-4}$ & 260 & 19 & $1.98 \times 10^{-3}$ & 518 \\
\hline 5 & 324 & $9.9 \times 10^{-4}$ & 30 & 10 & $6.15 \times 10^{-3}$ & 450 & 19 & $1.11 \times 10^{-2}$ & 819 \\
\hline 6 & 1156 & $6.4 \times 10^{-3}$ & 61 & 10 & $4.90 \times 10^{-2}$ & 879 & 19 & $9.01 \times 10^{-2}$ & 1606 \\
\hline 7 & 4356 & $5.7 \times 10^{-2}$ & 121 & 26 & $9.95 \times 10^{-1}$ & 4485 & 51 & 1.92 & 8578 \\
\hline 8 & 16900 & 0.6 & 234 & 3000 & 864.244 & 983107 & - & $>15 \mathrm{~min}$ & - \\
\hline
\end{tabular}

Table 6: Comparison between the OMP, MPS, and ADS methods.

functions. The results are indicated in Tables 7, 8 and 9 for different size of overlaps. The results show again that the OMP method is significantly faster than the ADS and MPS methods. Since the ADS and MPS methods fail to converge after some refinement steps, due to the degenerate 
overlap (with $p=4$ ), it is not possible to compute the time consumption in this case. The ADS method seems to behave worse than the MPS method with that respect. However, the performance of the OMP method, even for degenerate overlaps, is not compromised as we obtain the optimal convergence rate in all cases, and the computational cost is computed.

The MPS and ADS systems are symmetric. One can solve those systems using a CG solver. This would be two times faster. However, according to the observed results, the OMP method is still significantly faster.

\begin{tabular}{|c|c|c|c|c|c|c|c|c|c|}
\hline \multicolumn{10}{|c|}{ refinement level $=7$} \\
\hline \multicolumn{10}{|c|}{ Overlap size $=$ Degenerate } \\
\hline & & \multicolumn{2}{|c|}{ OMP Method } & \multicolumn{3}{|c|}{ MPS Method } & \multicolumn{3}{|c|}{ ADS Method } \\
\hline degree & \#dofs & time & \#it & calls & time & \#it & calls & time & $\#$ it \\
\hline$\overline{2}$ & 4356 & $8.2 \times 10^{-2}$ & 159 & 1490 & 50.2 & 229183 & 2799 & 93.8 & 430450 \\
\hline 3 & 4489 & $1.2 \times 10^{-1}$ & 131 & 395 & 22.4188 & 52090 & 898 & 50.6409 & 118263 \\
\hline 4 & 4624 & $9.5 \times 10^{-1}$ & 482 & - & - & - & - & - & - \\
\hline
\end{tabular}

Table 7: Comparison between the OMP, MPS and ADS methods for different degrees of the basis functions.

\begin{tabular}{|r|r||r|r||r|r|r||r|r|r|}
\hline \multicolumn{10}{|c|}{ refinement level $=7$} \\
\hline \multicolumn{10}{|c|}{ Overlap size $=$ Small } \\
\hline degree & \#dofs & OMP Method & \multicolumn{3}{|c|}{ MPS Method } & \multicolumn{3}{|c|}{ ADS Method } \\
\hline 2 & 4356 & $6.1 \times 10^{-2}$ & 128 & 52 & 1.7 & 8090 & 121 & 4.07 & 18630 \\
3 & 4489 & $1.1 \times 10^{-1}$ & 109 & 58 & 3.45672 & 7836 & 111 & 6.41868 & 14882 \\
4 & 4624 & $1.8 \times 10^{-1}$ & 104 & 38 & 3.25108 & 4766 & 74 & 6.28625 & 9157 \\
\hline
\end{tabular}

Table 8: Comparison between the OMP, MPS and ADS methods for different degrees of the basis functions.

\begin{tabular}{|c|c|c|c|c|c|c|c|c|c|}
\hline \multicolumn{10}{|c|}{ refinement level $=7$} \\
\hline \multicolumn{10}{|c|}{ Overlap size $=$ large } \\
\hline & & \multicolumn{2}{|c|}{ OMP Method } & \multicolumn{3}{|c|}{ MPS Method } & \multicolumn{3}{|c|}{ ADS Method } \\
\hline degree & \#dofs & time & \#it & calls & time & \#it & calls & time & $\#$ it \\
\hline 2 & 4356 & $5.7 \times 10^{-2}$ & 121 & 26 & $9.95 \times 10^{-1}$ & 4485 & 51 & 1.92 & 8578 \\
\hline 3 & 4489 & $9.5 \times 10^{-2}$ & 104 & 25 & 1.62 & 3684 & 44 & 2.80 & 6397 \\
\hline 4 & 4624 & 0.15 & 94 & 16 & 1.53 & 2194 & 22 & 2.07 & 2963 \\
\hline
\end{tabular}

Table 9: Comparison between the OMP, MPS and ADS methods for different degrees of the basis functions.

\section{Conclusion}

We proposed the OMP method, which is an isogeometric method for the numerical simulation on overlapping multi-patch structures, where the individual patches are represented by tensor-product 
spline parameterizations. We observe that neither trimming nor reparameterization are required for domains constructed by Boolean unions. Also, the OMP method avoids the iterative approach of the ADS or MPS methods.

The coupling of the individual solutions on the subdomains relies on two different extension operators, CEO and QEO. We explored that their convergence rates behave analogously. It should be noted that we cannot guarantee the uniqueness of the solution with respect to CEO (not even numerically), while numerical experiments indicate that coercivity of the discrete formulation is satisfied for QEO. Instabilities due to the lack of coercivity for CEO may be possible but were never observed. In all examples we obtain optimal convergence rates and did not experience any stability issues.

We compared the OMP method with the additive and multiplicative Schwarz methods, with respect to computation time. While ADS and MPS are not efficient for small overlaps, the OMP method provides the optimal rate of convergence even in these situations. Moreover, we can employ OMP to more than two patches with pairwise overlaps and in any dimension.

In our ongoing work, we plan to extend the OMP method to multi-patch domains containing overlaps of three or more patches.

Furthermore, we are interested in studying higher order PDEs, where a higher order of smoothness needs to be imposed on the boundary of the overlaps. Another possible extension is the case of PDEs on surfaces. We believe that the use of overlapping multi-patch structures might be useful for applications in geometric modeling as well. As an additional difficulty, the individual patches may not coincide exactly in the overlap, but only approximately.

The OMP method consists of a single monolithic linear system that needs to be solved. This is different from the additive Schwarz methods, which are inherently iterative and parallelizable. Nevertheless, existing and well developed packages for parallelizing large sparse linear systems (e.g. Trilinos or Hypre) can be employed to obtain a parallel version of our method. The structure of the global matrix can be exploited by splitting into sub-problems. Devising an adapted parallel version of our method is also a potential topic for future research.

\section{Acknowledgments}

This work was supported by the ERC AdG project CHANGE n. 694515, by the Austrian Science Fund (FWF) through project NFN S11708 as well as by the FWF together with the government of Upper Austria through the project P 30926-NBL. This support is gratefully acknowledged.

\section{References}

[1] T. J. R. Hughes, J. Cottrell, Y. Bazilevs, Isogeometric analysis: CAD, finite elements, NURBS, exact geometry and mesh refinement, Computer Methods in Applied Mechanics and Engineering 194 (39-41) (2005) 4135-4195. 
[2] J. A. Cottrell, T. J. R. Hughes, Y. Bazilevs, Isogeometric Analysis: Toward Integration of CAD and FEA, John Wiley and Sons, Ltd, 2009.

[3] A. Buffa, G. Sangalli (Eds.), IsoGeometric Analysis: A New Paradigm in the Numerical Approximation of PDEs, Lecture Notes in Mathematics, Springer International Publishing Switzerland, 2016.

[4] L. Beirão Da Veiga, A. Buffa, G. Sangalli, R. Vázquez, Mathematical analysis of variational isogeometric methods, Acta Numerica 23 (2014) 157-287.

[5] B. Marussig, T. J. R. Hughes, A review of trimming in isogeometric analysis: Challenges, data exchange and simulation aspects, Archives of Computational Methods in Engineering 25 (2018) 1059-1127.

[6] M. Scott, D. Thomas, E. Evans, Isogeometric spline forests, Computer Methods in Applied Mechanics and Engineering 269 (2014) 222-264.

[7] F. Buchegger, B. Jüttler, Planar multi-patch domain parameterization via patch adjacency graphs, Computer-Aided Design 82 (2017) 2-12.

[8] S. K. Kleiss, C. Pechstein, B. B. Jüttler, S. Tomar, IETI-isogeometric tearing and interconnecting, Computer Methods in Applied Mechanics and Engineering 247 (2012) 201-215.

[9] F. Buchegger, B. Jüttler, Adaptively refined multi-patch B-splines with enhanced smoothness, Applied Mathematics and Computation 272 (2016) 159-172.

[10] A. Collin, G. Sangalli, T. Takacs, Analysis-suitable $G^{1}$ multi-patch parametrizations for $C^{1}$ isogeometric spaces, Computer Aided Geometric Design 47 (2016) 93-113.

[11] M. Kapl, G. Sangalli, T. Takacs, Construction of analysis-suitable $G^{1}$ planar multi-patch parameterizations, Computer-Aided Design 97 (2018) 41-55.

[12] E. Brivadis, A. Buffa, B. Wohlmuth, L. Wunderlich, Isogeometric mortar methods, Computer Methods in Applied Mechanics and Engineering 284 (2015) 292-319.

[13] S. Deparis, D. Forti, P. Gervasio, A. Quarteroni, INTERNODES: an accurate interpolationbased method for coupling the galerkin solutions of PDEs on subdomains featuring nonconforming interfaces, Computers and Fluids 141 (2016) 22-41.

[14] P. Gervasioa, A. Quarteroni, Analysis of the INTERNODES method for non-conforming discretizations of elliptic equations, Computer Methods in Applied Mechanics and Engineering 334 (2018) 138-166. 
[15] A. Apostolatos, R. Schmidt, R. Wüchner, K.-U. Bletzinger, A Nitsche-type formulation and comparison of the most common domain decomposition methods in isogeometric analysis, International Journal for Numerical Methods in Engineering 97 (7) (2014) 473-504.

[16] V. P. Nguyen, P. Kerfriden, M. Brino, S. P. Bordas, E. Bonisoli, Nitsche's method for two and three dimensional NURBS patch coupling, Computational Mechanics 53 (6) (2014) 1163-1182.

[17] Y. Guo, M. Ruess, Nitsche's method for a coupling of isogeometric thin shells and blended shell structures, Computer Methods in Applied Mechanics and Engineering 284 (2015) 881-905.

[18] M. Ruess, D. Schillinger, A. I. Özcan, E. Rank, Weak coupling for isogeometric analysis of non-matching and trimmed multi-patch geometries, Computer Methods in Applied Mechanics and Engineering 269 (2014) 46-71.

[19] R. Bouclier, J.-C. Passieux, A Nitsche-based non-intrusive coupling strategy for global/local isogeometric structural analysis, Computer Methods in Applied Mechanics and Engineering 340 (2018) 253-277.

[20] F. Brunero, Discontinuous Galerkin methods for isogeometric analysis, Master's thesis, Università degli Studi di Milano (2012).

[21] U. Langer, A. Mantzaflaris, S. E. Moore, I. Toulopoulos, Multipatch discontinuous Galerkin isogeometric analysis, Vol. 107, Springer, 2015, pp. 1-32.

[22] C. Hofer, U. Langer, Dual-primal isogeometric tearing and interconnecting solvers for multipatch dG-IgA equations, Computer Methods in Applied Mechanics and Engineering 316 (2017) $2-21$.

[23] F. Massarwi, G. Elber, A B-spline based framework for volumetric object modeling, ComputerAided Design 78 (2016) 36-47.

[24] H.-J. Kim, Y.-D. Seo, S.-K. Youn, Isogeometric analysis for trimmed CAD surfaces, Computer Methods in Applied Mechanics and Engineering 198 (37-40) (2009) 2982-2995.

[25] R. Schmidt, R. Wüchner, K.-U. Bletzinger, Isogeometric analysis of trimmed NURBS geometries, Computer Methods in Applied Mechanics and Engineering 241 (2012) 93-111.

[26] K. Höllig, Finite Element Methods with B-Splines, Vol. 26, SIAM, 2003.

[27] R. Sanches, P. Bornemann, F.Cirak, Immersed B-spline (i-spline) finite element method for geometrically complex domains, Computer Methods in Applied Mechanics and Engineering 200 (2011) 1432-1445. 
[28] B. Marussig, R. Hiemstra, T. J. R. Hughes, Improved conditioning of isogeometric analysis matrices for trimmed geometries, Computer Methods in Applied Mechanics and Engineering 334 (2018) 79-110.

[29] D. Schillinger, M. Ruess, N. Zander, Y. Bazilevs, A. Düster, E. Rank, Small and large deformation analysis with the p-and B-spline versions of the Finite Cell Method, Computational Mechanics 50 (2012) 445-478.

[30] A. Düster, J. Parvizian, Z. Yang, E. Rank, The Finite Cell Method for three-dimensional problems of solid mechanics, Computer Methods in Applied Mechanics and Engineering 197 (2008) 3768-3782.

[31] L. Kudela, N. Zander, S. Kollmannsberger, E. Rank, Smart octrees: Accurately integrating discontinuous functions in 3D, Computer Methods in Applied Mechanics and Engineering 306 (2016) 406-426.

[32] A. P. Nagy, D. J. Benson, On the numerical integration of trimmed isogeometric elements, Computer Methods in Applied Mechanics and Engineering 284 (2015) 165-185.

[33] F. Scholz, A. Mantzaflaris, B. Jüttler, First order error correction for trimmed quadrature in isogeometric analysis, in: Advanced Finite Element Methods with Applications, Springer, 2019, pp. 297-321.

[34] M. Breitenberger, A. Apostolatos, B. Philipp, R. Wuechner, K. Bletzinger, Analysis in Computer Aided Design: Nonlinear isogeometric B-Rep analysis of shell structures, Computer Methods in Applied Mechanics and Engineering 284 (2015) 401-457.

[35] H. Zhang, R. Mo, N. Wan, An IGA discontinuous Galerkin method on the union of overlapped patches, Computer Methods in Applied Mechanics and Engineering 326 (2017) 445-418.

[36] F. Massarwi, B. van Sosin, G. Elber, Untrimming: Precise conversion of trimmed-surfaces to tensor-product surfaces, Computers \& Graphics 70 (2018) 80-91.

[37] M. Randrianarivony, Geometric processing of CAD data and meshes as input of integral equation solvers, Ph.D. thesis, Computer Science Faculty, Technische Universität Chemnitz (2006).

[38] S. Xia, X. Wang, X. Qian, Continuity and convergence in rational triangular Bézier spline based isogeometric analysis, Computer Methods in Applied Mechanics and Engineering 297 (2015) 292-324.

[39] X. Cai, Overlapping Domain Decomposition Methods, Vol. 33, Springer and Berlin and Heidelberg, 2003. 
[40] M. Bercovier, I. Soloveichik, Overlapping non matching meshes domain decomposition method in isogeometric analysis, arXiv 1502.03756v1.

[41] H. Prautzsch, W. Boehm, M. Paluszny, Bézier and B-Spline Techniques, Springer, 2002.

[42] J. Monterde, H. Ugail, On harmonic and biharmonic Bézier surfaces, Computer Aided Geometric Design 21 (2004) 697-715.

[43] A. Mantzaflaris, S. Kargaran, others (see website), G+smo (geometry plus simulation modules) v0.8.1, http://github.com/gismo (2018).

[44] F. Auricchio, L. Beirão da Veiga, A. Buffa, C. Lovadina, A. Reali, G. Sangalli, A fully "lockingfree" isogeometric approach for plane linear elasticity problems: A stream function formulation, Computer Methods in Applied Mechanics and Engineering 197 (2007) 160-172.

[45] L. Beirão Da Veiga, D. Cho, L. F. Pavarino, S. Scacchi, Overlapping Schwarz methods for isogeometric analysis, SIAM Journal on Numerical Analysis 50 (2012) 1394-1416.

[46] V. Dolean, P. Jolivet, F. Nataf, An Introduction to Domain Decomposition Methods: Algorithms, Theory, and Parallel Implementation, SIAM, 2015.

[47] L. Beirão Da Veiga, D. Cho, L. F. Pavarino, S. Scacchi, Isogeometric Schwarz preconditioners for linear elasticity systems, Computer Methods in Applied Mechanics and Engineering 253 (2014) 439-454.

[48] L. Beirão Da Veiga, D. Cho, L. F. Pavarino, S. Scacchi, Overlapping schwarz preconditioners for isogeometric collocation methods, Computer Methods in Applied Mechanics and Engineering 278 (2014) 239-253.

\section{Appendix A. Proof of Theorem 1}

First we prove (i). The assumption (4) about the extension operators implies that the two functions $u_{0}^{k}=\left.u\right|_{\Omega^{k}}-M^{k}\left(\left.u\right|_{\Omega^{k^{\prime}}}\right), k=1,2$, belong to $H_{0}^{1}\left(\Omega^{k}\right)$. In order to verify that $\left(u_{0}^{1}, u_{0}^{2}\right)$ satisfies the localized weak form of the Poisson problem, we introduce the trivial extension operators $T^{k}: H_{0}^{1}\left(\Omega^{k}\right) \rightarrow H_{0}^{1}(\Omega)$,

$$
T^{k} v^{k}=\left\{\begin{array}{l}
v^{k} \text { on } \Omega^{k} \\
0 \text { on } \Omega \backslash \Omega^{k}
\end{array}\right.
$$


and note that $a^{k}\left(u, v^{k}\right)=a\left(u, T^{k} v^{k}\right)$ and $\ell^{k}\left(v^{k}\right)=\ell\left(T^{k} v^{k}\right)$. Therefore we evaluate the left-hand side of Eq. (8) for $u_{0}^{k}=\left.u\right|_{\Omega^{k}}-\left.M^{k} u\right|_{\Omega^{k^{\prime}}}$ :

$$
\begin{gathered}
a^{1}\left(M^{12}\left(\left.u\right|_{\Omega^{1}}-\left.M^{1} u\right|_{\Omega^{2}}+M^{1}\left(\left.u\right|_{\Omega^{2}}-\left.M^{2} u\right|_{\Omega^{1}}\right)\right), v^{1}\right)+ \\
a^{2}\left(M^{21}\left(\left.u\right|_{\Omega^{2}}-\left.M^{2} u\right|_{\Omega^{1}}+M^{2}\left(\left.u\right|_{\Omega^{1}}-\left.M^{1} u\right|_{\Omega^{2}}\right)\right), v^{2}\right)= \\
a^{1}\left(M^{12}\left(\left.u\right|_{\Omega^{1}}-M^{1} M^{2}\left(\left.u\right|_{\Omega^{1}}\right)\right), v^{1}\right)+a^{2}\left(M^{21}\left(\left.u\right|_{\Omega^{2}}-M^{2} M^{1}\left(\left.u\right|_{\Omega^{2}}\right)\right), v^{2}\right)= \\
a^{1}\left(M^{12}\left(I^{1}-M^{1} M^{2}\right)\left(\left.u\right|_{\Omega^{1}}\right), v^{1}\right)+a^{2}\left(M^{21}\left(I^{2}-M^{2} M^{1}\right)\left(\left.u\right|_{\Omega^{2}}\right), v^{2}\right)= \\
a^{1}\left(\left.u\right|_{\Omega^{1}}, v^{1}\right)+a^{2}\left(\left.u\right|_{\Omega^{2}}, v^{2}\right),
\end{gathered}
$$

because $M^{k k^{\prime}}\left(I^{k}-M^{k} M^{k^{\prime}}\right)^{-1}=I$. We use the trivial extension operators and the assumption that $u$ solves the weak form (2) of the problem to rewrite the result as

$$
a^{1}\left(\left.u\right|_{\Omega^{1}}, v^{1}\right)+a^{2}\left(\left.u\right|_{\Omega^{2}}, v^{2}\right)=a\left(\left.u\right|_{\Omega^{1}}, T^{1} v^{1}\right)+a\left(\left.u\right|_{\Omega^{2}}, T^{2} v^{2}\right)=\ell\left(T^{1} v^{1}\right)+\ell\left(T^{2} v^{2}\right)=\ell^{1}\left(v^{1}\right)+\ell^{2}\left(v^{2}\right),
$$

thereby completing the proof of (i).

Second we prove (ii). We consider the restrictions of the two forms

$$
a^{12}\left(u^{12}, v^{12}\right)=\int_{\Phi^{12}} \nabla u^{12} \nabla v^{12} \mathrm{~d} \xi \quad \text { and } \quad \ell^{12}\left(v^{12}\right)=\int_{\Phi^{12}} f v^{12} \mathrm{~d} \xi
$$

to the overlap region $\Phi^{12}$ and the trivial extension operator $T^{12}: H_{0}^{1}\left(\Phi^{12}\right) \rightarrow H_{0}^{1}(\Omega)$,

$$
T^{12} v^{12}=\left\{\begin{array}{l}
v^{12} \text { on } \Phi^{12} \\
0 \text { on } \Omega \backslash \Phi^{12}
\end{array}\right.
$$

We first show that $M^{12}\left(u_{0}^{1}+M^{1} u_{0}^{2}\right)$ and $M^{21}\left(u_{0}^{2}+M^{2} u_{0}^{1}\right)$ coincide on the boundary of $\Phi^{12}$. We know, $M^{k k^{\prime}}=\sum_{\ell=0}^{\infty}\left(M^{k} M^{k^{\prime}}\right)^{\ell}$, then we can write

$$
\begin{aligned}
& M^{12}\left(u_{0}^{1}+M^{1} u_{0}^{2}\right)=\sum_{\ell=0}^{\infty}\left(M^{1} M^{2}\right)^{\ell}\left(u_{0}^{1}+M^{1} u_{0}^{2}\right) \\
& M^{21}\left(u_{0}^{2}+M^{2} u_{0}^{1}\right)=\sum_{\ell=0}^{\infty}\left(M^{2} M^{1}\right)^{\ell}\left(u_{0}^{2}+M^{2} u_{0}^{1}\right) .
\end{aligned}
$$

According to the assumption (4) we get on $\Gamma_{C}^{1}$

$$
\sum_{\ell=0}^{\infty}\left(M^{1} M^{2}\right)^{\ell}\left(u_{0}^{1}+M^{1} u_{0}^{2}\right)=\sum_{\ell=0}^{\infty}\left(M^{1} M^{2}\right)^{\ell} u_{0}^{1}+\sum_{\ell=0}^{\infty}\left(M^{1} M^{2}\right)^{\ell} \underbrace{M^{1} u_{0}^{2}}_{=u_{0}^{2}} .
$$


The first sum satisfies

$$
\sum_{\ell=0}^{\infty}\left(M^{1} M^{2}\right)^{\ell} u_{0}^{1}=\underbrace{u_{0}^{1}}_{=0}+\sum_{\ell=1}^{\infty}\left(M^{1} M^{2}\right)^{\ell} u_{0}^{1}=\sum_{\ell=0}^{\infty}\left(M^{1} M^{2}\right)^{\ell} \underbrace{M^{1} M^{2} u_{0}^{1}}_{=M^{2} u_{0}^{1}},
$$

from which we obtain

$$
M^{12}\left(u_{0}^{1}+M^{1} u_{0}^{2}\right)=\sum_{\ell=0}^{\infty}\left(M^{1} M^{2}\right)^{\ell}\left(u_{0}^{2}+M^{2} u_{0}^{1}\right)=M^{21}\left(u_{0}^{2}+M^{2} u_{0}^{1}\right), \text { on } \Gamma_{C}^{1} .
$$

Analogously we get

$$
M^{21}\left(u_{0}^{2}+M^{2} u_{0}^{1}\right)=\sum_{\ell=0}^{\infty}\left(M^{2} M^{1}\right)^{\ell}\left(u_{0}^{1}+M^{1} u_{0}^{2}\right)=M^{12}\left(u_{0}^{1}+M^{1} u_{0}^{2}\right), \text { on } \Gamma_{C}^{2} .
$$

Therefore, the solutions are equal on the boundary of $\Phi^{12}$.

For any $v^{12} \in H_{0}^{1}\left(\Phi^{12}\right)$, we get

$$
a^{12}\left(M^{12}\left(u_{0}^{1}+M^{1} u_{0}^{2}\right), v^{12}\right)=a^{1}\left(M^{12}\left(u_{0}^{1}+M^{1} u_{0}^{2}\right), T^{12} v^{12}\right)=\ell^{1}\left(T^{12} v^{12}\right)=\ell^{12}\left(v^{12}\right)
$$

and

$$
a^{12}\left(M^{21}\left(u_{0}^{2}+M^{2} u_{0}^{1}\right), v^{12}\right)=a^{2}\left(M^{21}\left(u_{0}^{2}+M^{2} u_{0}^{1}\right), T^{12} v^{12}\right)=\ell^{2}\left(T^{12} v^{12}\right)=\ell^{12}\left(v^{12}\right),
$$

since $\left(u_{0}^{1}, u_{0}^{2}\right)$ solves Problem 2. Hence, we obtain

$$
a^{12}\left(M^{12}\left(u_{0}^{1}+M^{1} u_{0}^{2}\right)-M^{21}\left(u_{0}^{2}+M^{2} u_{0}^{1}\right), v^{12}\right)=0,
$$

for all $v^{12} \in H_{0}^{1}\left(\Phi^{12}\right)$. Since $M^{21}\left(u_{0}^{2}+M^{2} u_{0}^{1}\right)$ and $M^{21}\left(u_{0}^{2}+M^{2} u_{0}^{1}\right)$ coincide on the boundary of $\Phi^{12}$, we can conclude from the coercivity of $a(\cdot, \cdot)$ that

$$
M^{12}\left(u_{0}^{1}+M^{1} u_{0}^{2}\right)=M^{21}\left(u_{0}^{2}+M^{2} u_{0}^{1}\right) \text { on } \Phi^{12} .
$$

Any $v \in H_{0}^{1}(\Omega)$ can be represented as $v=T^{1} v^{1}+T^{2} v^{2}$, where $v^{k} \in H_{0}^{1}\left(\Omega^{k}\right)$. We thus obtain

$$
a(u, v)=a\left(u, T^{1} v^{1}+T^{2} v^{2}\right)=a^{1}\left(\left.u\right|_{\Omega^{1}}, v^{1}\right)+a^{2}\left(\left.u\right|_{\Omega^{2}}, v^{2}\right)
$$

and

$$
\ell(v)=\ell\left(T^{1} v^{1}+T^{2} v^{2}\right)=\ell^{1}\left(v^{1}\right)+\ell^{2}\left(v^{2}\right) .
$$


We have

$$
\left.u\right|_{\Omega^{k}}=M^{k k^{\prime}}\left(u_{0}^{k}+M^{k} u_{0}^{k^{\prime}}\right)
$$

by definition (10) for $k=1$ and due to (A.2) for $k=2$. Since $\left(u_{0}^{1}, u_{0}^{2}\right)$ solves Problem 2, we obtain

$$
\begin{aligned}
a(u, v) & =a^{1}\left(\left.u\right|_{\Omega^{1}}, v^{1}\right)+a^{2}\left(\left.u\right|_{\Omega^{2}}, v^{2}\right) \\
& =a^{1}\left(M^{12}\left(u_{0}^{1}+M^{1} u_{0}^{2}\right), v^{1}\right)+a^{2}\left(M^{21}\left(u_{0}^{2}+M^{2} u_{0}^{1}\right), v^{2}\right) \\
& =\ell^{1}\left(v^{1}\right)+\ell^{2}\left(v^{2}\right)=\ell(v) .
\end{aligned}
$$

This completes the proof of (ii).

Third we prove (iii). We consider two solutions $\left(u_{0}^{1}, u_{0}^{2}\right)$ and $\left(\tilde{u}_{0}^{1}, \tilde{u}_{0}^{2}\right)$ of Problem 2, which correspond to the same solution $u$ of Problem 1.

As we showed in (A.2) the solutions are equal on $\Phi^{12}$, so we can write

$$
u=\underbrace{M^{12}\left(u_{0}^{1}+M^{1} u_{0}^{2}\right)}_{A_{1}}=\underbrace{M^{21}\left(u_{0}^{2}+M^{2} u_{0}^{1}\right)}_{A_{2}}=\underbrace{M^{12}\left(\tilde{u}_{0}^{1}+M^{1} \tilde{u}_{0}^{2}\right)}_{A_{3}}=\underbrace{M^{21}\left(\tilde{u}_{0}^{2}+M^{2} \tilde{u}_{0}^{1}\right)}_{A_{4}} \text { on } \Phi^{12} .
$$

Since $A_{1}=A_{3}$ and $A_{2}=A_{4}$ we can write

$$
\begin{aligned}
& M^{12}\left(u_{0}^{1}+M^{1} u_{0}^{2}\right)=M^{12}\left(\tilde{u}_{0}^{1}+M^{1} \tilde{u}_{0}^{2}\right), \text { on } \Phi^{12} \\
& M^{21}\left(u_{0}^{2}+M^{2} u_{0}^{1}\right)=M^{21}\left(\tilde{u}_{0}^{2}+M^{2} \tilde{u}_{0}^{1}\right), \text { on } \Phi^{12} .
\end{aligned}
$$

We apply $\left(I-M^{1} M^{2}\right)$ and $\left(I-M^{2} M^{1}\right)$ to the first and second equations respectively and obtain

$$
\begin{aligned}
& \left(u_{0}^{1}-\tilde{u}_{0}^{1}\right)+M^{1}\left(u_{0}^{2}-\tilde{u}_{0}^{2}\right)=0, \text { on } \Phi^{12} \\
& \left(u_{0}^{2}-\tilde{u}_{0}^{2}\right)+M^{2}\left(u_{0}^{1}-\tilde{u}_{0}^{1}\right)=0, \text { on } \Phi^{12} .
\end{aligned}
$$

Now we apply $-M^{2}$ to the first equation and add both sides of the two equations and obtain

$$
\left(u_{0}^{2}-\tilde{u}_{0}^{2}\right)-M^{2} M^{1}\left(u_{0}^{2}-\tilde{u}_{0}^{2}\right)=0, \text { on } \Phi^{12}
$$

or equivalently

$$
\left(I-M^{2} M^{1}\right)\left(u_{0}^{2}-\tilde{u}_{0}^{2}\right)=0 .
$$

After applying $M^{21}$ to both sides of the last equation we obtain

$$
u_{0}^{2}=\tilde{u}_{0}^{2} \text { on } \Phi^{12} .
$$

Similarly, we conclude $u_{0}^{1}=\tilde{u}_{0}^{1}$ on $\Phi^{12}$. 
Moreover, we need to show that $u_{0}^{1}=\tilde{u}_{0}^{1}$ on $\Omega^{1} \backslash \Phi^{12}$ and $u_{0}^{2}=\tilde{u}_{0}^{2}$ on $\Omega^{2} \backslash \Phi^{12}$. We have

$$
a^{1}\left(M^{12}\left(u_{0}^{1}+M^{1} u_{0}^{2}\right), v_{0}^{1}\right)=\ell^{1}\left(v_{0}^{1}\right) \quad \text { and } \quad a^{1}\left(M^{12}\left(\tilde{u}_{0}^{1}+M^{1} \tilde{u}_{0}^{2}\right), v_{0}^{1}\right)=\ell^{1}\left(v_{0}^{1}\right),
$$

for all $v_{0}^{1} \in H_{0}^{1}\left(\Omega^{1}\right)$, which follows from (8) setting $v_{0}^{2}=0$ and (9). Taking the difference of the two equations, we obtain

$$
a^{1}\left(M^{12}\left(u_{0}^{1}-\tilde{u}_{0}^{1}+M^{1}\left(u_{0}^{2}-\tilde{u}_{0}^{2}\right)\right), v_{0}^{1}\right)=0 \quad \forall v_{0}^{1} \in H_{0}^{1}\left(\Omega^{1}\right) .
$$

According to Assumption 2 and (A.3), we have

$$
M^{1}\left(u_{0}^{2}-\tilde{u}_{0}^{2}\right)=0,
$$

so we are left with

$$
a^{1}\left(M^{12}\left(u_{0}^{1}-\tilde{u}_{0}^{1}\right), v_{0}^{1}\right)=0 \quad \forall v_{0}^{1} \in H_{0}^{1}\left(\Omega^{1}\right) .
$$

This is, by definition,

$$
a^{1}\left(\sum_{\ell=0}^{\infty}\left(M^{1} M^{2}\right)^{\ell}\left(u_{0}^{1}-\tilde{u}_{0}^{1}\right), v_{0}^{1}\right)=0 \quad \forall v_{0}^{1} \in H_{0}^{1}\left(\Omega^{1}\right) .
$$

For $\ell \geq 1$ we have $\left(M^{1} M^{2}\right)^{\ell}\left(u_{0}^{1}-\tilde{u}_{0}^{1}\right)=0$ due to Assumption 2. Hence, we get

$$
a^{1}\left(u_{0}^{1}-\tilde{u}_{0}^{1}, v_{0}^{1}\right)=0 \quad \forall v_{0}^{1} \in H_{0}^{1}\left(\Omega^{1}\right),
$$

from which we immediately obtain, due to the coercivity of the bilinear form $a^{1}$, that $u_{0}^{1}-\tilde{u}_{0}^{1}=0$ on $\Omega^{1}$. Analogously we obtain $u_{0}^{2}=\tilde{u}_{0}^{2}$ on the other subdomain $\Omega^{2}$, which completes the proof.

\section{Appendix B. Proof of Lemma 1}

We define the extension operator $M^{1}$ as

$$
M^{1} u^{2}(x)= \begin{cases}u^{2}\left(b_{1}\right)\left(\frac{x-a_{2}}{b_{1}-a_{2}}\right) & \text { in } \Phi^{12} \\ 0 & \text { otherwise }\end{cases}
$$

and $M^{2}$ analogously. We have $M^{1} u^{2}\left(a_{2}\right)=0$ and $M^{2} u^{1}\left(b_{1}\right)=0$ by definition. Hence, $M^{k} M^{k^{\prime}} u^{k} \equiv 0$ and consequently, $M^{1} u^{2}\left(b_{1}\right)=u_{0}^{2}\left(b_{1}\right)$ and $M^{2} u^{1}\left(a_{2}\right)=u_{0}^{1}\left(a_{2}\right)$.

We have

$$
\left|M^{1} u^{2}\right|_{H^{1}\left(\Omega^{1}\right)}^{2}=\int_{a_{1}}^{b_{1}}\left[\left(M^{1} u^{2}\right)^{\prime}\right]^{2} \mathrm{~d} x=\int_{a_{2}}^{b_{1}}\left[\frac{u_{0}^{2}\left(b_{1}\right)}{d}\right]^{2} \mathrm{~d} x=\frac{u_{0}^{2}\left(b_{1}\right)^{2}}{d},
$$






Figure B.14: 1D-patch setting.

where $d=b_{1}-a_{2}$. Analogously, $\left|M^{2} u^{1}\right|_{H^{1}\left(\Omega^{2}\right)}^{2}=\frac{u_{0}^{1}\left(a_{2}\right)^{2}}{d}$.

On the domain $\Omega^{1}$ we have

$$
\begin{aligned}
\left|u_{0}^{1}\left(a_{2}\right)\right|^{2} & =\lambda\left|\int_{a_{1}}^{a_{2}}\left(u_{0}^{1}\right)^{\prime} \cdot 1 \mathrm{~d} x\right|^{2}+(1-\lambda)\left|\int_{a_{2}}^{b_{1}}\left(u_{0}^{1}\right)^{\prime} \cdot 1 \mathrm{~d} x\right|^{2} \\
& \leq \lambda\left[\int_{a_{1}}^{a_{2}}\left(\left(u_{0}^{1}\right)^{\prime}\right)^{2} \mathrm{~d} x \int_{a_{1}}^{a_{2}} 1 \mathrm{~d} x\right]+(1-\lambda)\left[\int_{a_{2}}^{b_{1}}\left(\left(u_{0}^{1}\right)^{\prime}\right)^{2} \mathrm{~d} x \int_{a_{2}}^{b 1} 1 \mathrm{~d} x\right] \\
& =\lambda\left(a_{2}-a_{1}\right)\left|u_{0}^{1}\right|_{H^{1}\left(a_{1}, a_{2}\right)}^{2}+(1-\lambda)(d)\left|u_{0}^{1}\right|_{H^{1}\left(a_{2}, b 1\right)}^{2},
\end{aligned}
$$

where $\left(a_{2}-a_{1}\right)=\left|\Omega^{1}\right|-d$. For $\lambda=\frac{d}{\left|\Omega^{1}\right|}$ we obtain

$$
\left|u_{0}^{1}\left(a_{2}\right)\right| \leq \sqrt{d-\frac{d^{2}}{\left|\Omega^{1}\right|}} \cdot\left|u_{0}^{1}\right|_{H^{1}\left(\Omega^{1}\right)}
$$

and analogously for the second domain $\Omega^{2}$ we obtain

$$
\left|u_{0}^{2}\left(b_{1}\right)\right| \leq \sqrt{d-\frac{d^{2}}{\left|\Omega^{2}\right|}} \cdot\left|u_{0}^{2}\right|_{H^{1}\left(\Omega^{2}\right)} .
$$

According to (B.2), (B.3) and (B.4), we get

$$
\begin{aligned}
\left|M^{1} u^{2}\right|_{H^{1}\left(\Omega^{1}\right)} & \leq C_{1}\left|u_{0}^{2}\right|_{H^{1}\left(\Omega^{2}\right)} \\
\left|M^{2} u^{1}\right|_{H^{1}\left(\Omega^{2}\right)} & \leq C_{2}\left|u_{0}^{1}\right|_{H^{1}\left(\Omega^{1}\right)}
\end{aligned}
$$

where $C_{1}=\sqrt{1-\frac{d}{\left|\Omega^{2}\right|}}$ and $C_{2}=\sqrt{1-\frac{d}{\left|\Omega^{1}\right|}}$. 
- Boundedness of the bilinear form:

We have

$$
\begin{aligned}
\left|\mathcal{A}\left(\left(u^{1}, u^{2}\right),\left(v_{0}^{1}, v_{0}^{2}\right)\right)\right|= & \left|\int_{\Omega^{1}}\left(u_{0}^{1}+M^{1} u^{2}\right)^{\prime} v_{0}^{\prime 1} \mathrm{~d} x+\int_{\Omega^{2}}\left(u_{0}^{2}+M^{2} u^{1}\right)^{\prime} v_{0}^{\prime 2} \mathrm{~d} x\right| \\
\leq & \left|\int_{\Omega^{1}} u_{0}^{1^{\prime}} v_{0}^{1^{\prime}} \mathrm{d} x\right|+\left|\int_{\Omega^{1}}\left(M^{1} u^{2}\right)^{\prime} v_{0}^{1^{\prime}} \mathrm{d} x\right| \\
& +\left|\int_{\Omega^{2}} u_{0}^{2^{\prime}} v_{0}^{2^{\prime}} \mathrm{d} x\right|+\left|\int_{\Omega^{2}}\left(M^{2} u^{1}\right)^{\prime} v_{0}^{2^{\prime}} \mathrm{d} x\right| \\
& \leq\left|u_{0}^{1}\right|_{H^{1}\left(\Omega^{1}\right)}\left|v_{0}^{1}\right|_{H^{1}\left(\Omega^{1}\right)}+\left|M^{1} u^{2}\right|_{H^{1}\left(\Omega^{1}\right)}\left|v_{0}^{1}\right|_{H^{1}\left(\Omega^{1}\right)}+\left|u_{0}^{2}\right|_{H^{1}\left(\Omega^{2}\right)}\left|v_{0}^{2}\right|_{H^{1}\left(\Omega^{2}\right)} \\
& +\left|M^{2} u^{1}\right|_{H^{1}\left(\Omega^{2}\right)}\left|v_{0}^{2}\right|_{H^{1}\left(\Omega^{2}\right)},
\end{aligned}
$$

and according to (B.5)

$$
\begin{aligned}
\left|\mathcal{A}\left(\left(u^{1}, u^{2}\right),\left(v_{0}^{1}, v_{0}^{2}\right)\right)\right| & \leq\left|u_{0}^{1}\right|_{H^{1}\left(\Omega^{1}\right)}\left|v_{0}^{1}\right|_{H^{1}\left(\Omega^{1}\right)}+C_{1}\left|u_{0}^{2}\right|_{H^{1}\left(\Omega^{2}\right)}\left|v_{0}^{1}\right|_{H^{1}\left(\Omega^{1}\right)} \\
& +\left|u_{0}^{2}\right|_{H^{1}\left(\Omega^{2}\right)}\left|v_{0}^{2}\right|_{H^{1}\left(\Omega^{2}\right)}+C_{2}\left|u_{0}^{1}\right|_{H^{1}\left(\Omega^{1}\right)}\left|v_{0}^{2}\right|_{H^{1}\left(\Omega^{2}\right)} .
\end{aligned}
$$

Since $\|(\cdot, \cdot)\|_{H^{1}}=|(\cdot, \cdot)|_{H^{1}}+\|(\cdot, \cdot)\|_{L^{2}}$, we can write

$$
\begin{aligned}
\left|\mathcal{A}\left(\left(u^{1}, u^{2}\right),\left(v_{0}^{1}, v_{0}^{2}\right)\right)\right| \leq & \\
\| & u_{0}^{1}\left\|_{H^{1}\left(\Omega^{1}\right)}\right\| v_{0}^{1}\left\|_{H^{1}\left(\Omega^{1}\right)}+\right\| u_{0}^{2}\left\|_{H^{1}\left(\Omega^{2}\right)}\right\| v_{0}^{2} \|_{H^{1}\left(\Omega^{2}\right)} \\
& +C_{1}\left\|u_{0}^{2}\right\|_{H^{1}\left(\Omega^{2}\right)}\left\|v_{0}^{1}\right\|_{H^{1}\left(\Omega^{1}\right)}+C_{2}\left\|u_{0}^{1}\right\|_{H^{1}\left(\Omega^{1}\right)}\left\|v_{0}^{2}\right\|_{H^{1}\left(\Omega^{2}\right)} \\
& \leq C_{\max }\left(\left\|u_{0}^{1}\right\|_{H^{1}\left(\Omega^{1}\right)}+\left\|u_{0}^{2}\right\|_{H^{1}\left(\Omega^{2}\right)}\right)\left(\left\|v_{0}^{1}\right\|_{H^{1}\left(\Omega^{1}\right)}+\left\|v_{0}^{2}\right\|_{H^{1}\left(\Omega^{2}\right)}\right) \\
& \leq C_{\max } \sqrt{2\left(\left\|u_{0}^{1}\right\|_{H^{1}\left(\Omega^{1}\right)}^{2}+\left\|u_{0}^{2}\right\|_{H^{1}\left(\Omega^{2}\right)}^{2}\right)} \sqrt{2\left(\left\|v_{0}^{1}\right\|_{H^{1}\left(\Omega^{1}\right)}^{2}+\left\|v_{0}^{2}\right\|_{H^{1}\left(\Omega^{2}\right)}^{2}\right)} \\
= & \bar{\alpha}\left\|\left(u_{0}^{1}, u_{0}^{2}\right)\right\|_{H^{1}\left(\Omega^{1}\right) \times H^{1}\left(\Omega^{2}\right)}\left\|\left(v_{0}^{1}, v_{0}^{2}\right)\right\|_{H^{1}\left(\Omega^{1}\right) \times H^{1}\left(\Omega^{2}\right)},
\end{aligned}
$$

where $C_{\max }=\max \left\{1, C_{1}, C_{2}\right\}$ and $\bar{\alpha}=2 C_{\max }$. Therefore, the bilinear form is bounded.

- Boundedness of the linear form:

We have

$$
\begin{aligned}
\left|\mathcal{L}\left(\left(v_{0}^{1}, v_{0}^{2}\right)\right)\right|= & \left|\int_{\Omega^{1}} f v_{0}^{1} d \xi+\int_{\Omega^{2}} f v_{0}^{2} d \xi\right| \\
& \leq \Lambda_{1}\left(\left\|v_{0}^{1}\right\|_{L^{2}\left(\Omega^{1}\right)}+\left\|v_{0}^{2}\right\|_{L^{2}\left(\Omega^{2}\right)}\right) \\
& \leq \Lambda_{1}\left(\left\|v_{0}^{1}\right\|_{H^{1}\left(\Omega^{1}\right)}+\left\|v_{0}^{2}\right\|_{H^{1}\left(\Omega^{2}\right)}\right) \\
& \leq \sqrt{2} \Lambda_{1} \sqrt{\left\|v_{0}^{1}\right\|_{H^{1}\left(\Omega^{1}\right)}^{2}+\left\|v_{0}^{2}\right\|_{H^{1}\left(\Omega^{2}\right)}^{2}} \\
& =\Lambda\left\|\left(v_{0}^{1}, v_{0}^{2}\right)\right\|_{H^{1}\left(\Omega^{1}\right) \times H^{1}\left(\Omega^{2}\right)},
\end{aligned}
$$


where $\Lambda=\sqrt{2} \Lambda_{1}$. Therefore, the linear form is bounded.

- Coercivity of the bilinear form:

According to (B.5) we obtain

$$
\begin{aligned}
\mathcal{A}\left(\left(u^{1}, u^{2}\right),\left(u_{0}^{1}, u_{0}^{2}\right)\right)= & \int_{\Omega^{1}}\left(u_{0}^{1}+M^{1} u^{2}\right)^{\prime} u_{0}^{1^{\prime}}+\int_{\Omega^{2}}\left(u_{0}^{2}+M^{2} u^{1}\right)^{\prime} u_{0}^{2^{\prime}} \\
= & \int_{\Omega^{1}}\left(u_{0}^{1}\right)^{\prime}\left(u_{0}^{1}\right)^{\prime}+\int_{\Omega^{2}}\left(u_{0}^{2}\right)^{\prime}\left(u_{0}^{2}\right)^{\prime}+\int_{\Omega^{1}}\left(M^{1} u^{2}\right)^{\prime}\left(u_{0}^{1}\right)^{\prime}+\int_{\Omega^{2}}\left(M^{2} u^{1}\right)^{\prime}\left(u_{0}^{2}\right)^{\prime} \\
& \geq \int_{\Omega^{1}}\left(u_{0}^{1}\right)^{\prime}\left(u_{0}^{1}\right)^{\prime}+\int_{\Omega^{2}}\left(u_{0}^{2}\right)^{\prime}\left(u_{0}^{2}\right)^{\prime}-\left|\int_{\Omega^{1}}\left(M^{1} u^{2}\right)^{\prime}\left(u_{0}^{1}\right)^{\prime}+\int_{\Omega^{2}}\left(M^{2} u^{1}\right)^{\prime}\left(u_{0}^{2}\right)^{\prime}\right| \\
& \geq\left|u_{0}^{1}\right|_{H^{1}\left(\Omega^{1}\right)}^{2}+\left|u_{0}^{2}\right|_{H^{1}\left(\Omega^{2}\right)}^{2}-\left|M^{1} u^{2}\right|_{H^{1}\left(\Omega^{1}\right)}\left|u_{0}^{1}\right|_{H^{1}\left(\Omega^{1}\right)}-\left|M^{2} u^{1}\right|_{H^{1}\left(\Omega^{2}\right)}\left|u_{0}^{2}\right|_{H^{1}\left(\Omega^{2}\right)} .
\end{aligned}
$$

Therefore, according to (B.5) we can write

$$
\begin{aligned}
\mathcal{A}\left(\left(u^{1}, u^{2}\right),\left(u_{0}^{1}, u_{0}^{2}\right)\right) & \geq\left|u_{0}^{1}\right|_{H^{1}\left(\Omega^{1}\right)}^{2}+\left|u_{0}^{2}\right|_{H^{1}\left(\Omega^{2}\right)}^{2}-\left(C_{1}+C_{2}\right)\left|u_{0}^{1}\right|_{H^{1}\left(\Omega^{1}\right)}\left|u_{0}^{2}\right|_{H^{1}\left(\Omega^{2}\right)} \\
& \geq\left|u_{0}^{1}\right|_{H^{1}\left(\Omega^{1}\right)}^{2}+\left|u_{0}^{2}\right|_{H^{1}\left(\Omega^{2}\right)}^{2}-\frac{1}{2}\left(C_{1}+C_{2}\right)\left(\left|u_{0}^{1}\right|_{H^{1}\left(\Omega^{1}\right)}^{2}+\left|u_{0}^{2}\right|_{H^{1}\left(\Omega^{2}\right)}^{2}\right) \\
& =\left(1-\frac{1}{2}\left(C_{1}+C_{2}\right)\right)\left(\left|u_{0}^{1}\right|_{H^{1}\left(\Omega^{1}\right)}^{2}+\left|u_{0}^{2}\right|_{H^{1}\left(\Omega^{2}\right)}^{2}\right) \\
& \geq \eta\left(1-\frac{1}{2}\left(C_{1}+C_{2}\right)\right)\left(\left\|u_{0}^{1}\right\|_{H^{1}\left(\Omega^{1}\right)}^{2}+\left\|u_{0}^{2}\right\|_{H^{1}\left(\Omega^{2}\right)}^{2}\right) \\
& =\underline{\alpha}\left\|\left(u_{0}^{1}, u_{0}^{2}\right)\right\|_{H^{1}\left(\Omega^{1}\right) \times H^{1}\left(\Omega^{2}\right)}^{2},
\end{aligned}
$$

where $\eta$ is the constant from the Poincaré inequality and $\underline{\alpha}=\eta\left(1-\frac{1}{2} \sqrt{1-\frac{d}{\left|\Omega^{2}\right|}}-\frac{1}{2} \sqrt{1-\frac{d}{\left|\Omega^{1}\right|}}\right)$. This shows the coercivity of the bilinear form and concludes the proof. Note that for a fixed two patch domain this constant is always positive and bounded away from zero, since $0<\frac{d}{\left|\Omega^{k}\right|}<1$. However, if the overlap degenerates, the constant $\underline{\alpha}$ goes to zero. 